\title{
Wnt11 regulates cardiac chamber development and disease during perinatal maturation
}

\author{
Marlin Touma, ${ }^{1,2}$ Xuedong Kang, ${ }^{1,2}$ Fuying Gao, ${ }^{3}$ Yan Zhao, ${ }^{1,2}$ Ashley A. Cass, ${ }^{4}$ Reshma Biniwale, ${ }^{5}$ \\ Xinshu Xiao, ${ }^{4}$ Mansuoreh Eghbali, ${ }^{6}$ Giovanni Coppola, ${ }^{3}$ Brian Reemtsen, ${ }^{5}$ and Yibin Wang ${ }^{2,7}$ \\ 'The Children's Discovery and Innovation Institute (CDI), Department of Pediatrics, '2Cardiovascular Research Laboratory, \\ ${ }^{3}$ Department of Neurology, ${ }^{4}$ Bioinformatics Interdepartmental Program, ${ }^{5}$ Department of Cardiothoracic Surgery, \\ ${ }^{6}$ Department of Anesthesiology, and 'Department of Anesthesiology, Physiology and Medicine, University of California, \\ Los Angeles, California, USA.
}

Ventricular chamber growth and development during perinatal circulatory transition is critical for functional adaptation of the heart. However, the chamber-specific programs of neonatal heart growth are poorly understood. We used integrated systems genomic and functional biology analyses of the perinatal chamber specific transcriptome and we identified Wnt11 as a prominent regulator of chamber-specific proliferation. Importantly, downregulation of Wnt11 expression was associated with cyanotic congenital heart defect (CHD) phenotypes and correlated with $\mathrm{O}_{2}$ saturation levels in hypoxemic infants with Tetralogy of Fallot (TOF). Perinatal hypoxia treatment in mice suppressed Wnt11 expression and induced myocyte proliferation more robustly in the right ventricle, modulating Rb1 protein activity. Wnt11 inactivation was sufficient to induce myocyte proliferation in perinatal mouse hearts and reduced Rb1 protein and phosphorylation in neonatal cardiomyocytes. Finally, downregulated Wnt11 in hypoxemic TOF infantile hearts was associated with Rb1 suppression and induction of proliferation markers. This study revealed a previously uncharacterized function of Wnt11-mediated signaling as an important player in programming the chamber-specific growth of the neonatal heart. This function influences the chamber-specific development and pathogenesis in response to hypoxia and cyanotic CHDs. Defining the underlying regulatory mechanism may yield chamber-specific therapies for infants born with CHDs.

Conflict of interest: The authors have declared that no conflict of interest exists.

Submitted: May 5, 2017

Accepted: August 3, 2017

Published: September 7, 2017

Reference information:

JCI Insight. 2017;2(17):e94904.

https://doi.org/10.1172/jci.

insight. 94904.

\section{Introduction}

The embryologic origins of the right ventricle (RV) and the left ventricle (LV) are different (1-5), preparing them to perform different tasks after birth. During fetal development, the RV assumes the dominant pump function. At birth, perinatal circulatory transition leads to increased oxygenation and separation of the pulmonary and the systemic circulation rapidly when the LV assumes the dominant pump function (1-3). Concomitant with morphological and functional modifications in the LV and RV $(4,5)$, the neonatal cardiomyocytes (CMCs) undergo dramatic changes in morphology, respiration, metabolism, contractile function, and withdraw from the cell cycle (6-13). It is known that distinct key transcription factors drive the chamber-specific gene networks during development $(14,15)$. However, the molecular network involved in regulating the differential LV-versus-RV myocardial growth in the postnatal heart is less defined.

Remote developmental signals, dynamic hemodynamic changes, altered metabolic demands, and different environmental stressors may impact the chamber-specific growth and development programs, especially in the context of congenital heart defects (CHDs). The altered anatomy of a CHD heart can be further complicated by the persistence of fetal shunt pathways, pathological flow patterns and hypoxemia (16-18). As such, the growing heart is particularly vulnerable to multi-factorial perinatal stresses. Hypoxia is a major and commonly encountered stress factor that may delay perinatal circulatory transition $(2,3)$. On the other hand, recent evidence has also demonstrated that hypoxia induces CMC cell cycle reentry and cardiac regeneration $(19,20)$. Lacking the mechanistic resolution, our current approaches to management of failing heart with a CHD have not been fully effective. Consequently, infants born with CHDs, particularly those with critical forms of CHDs are at increased risk especially within the first few weeks of life. 
A

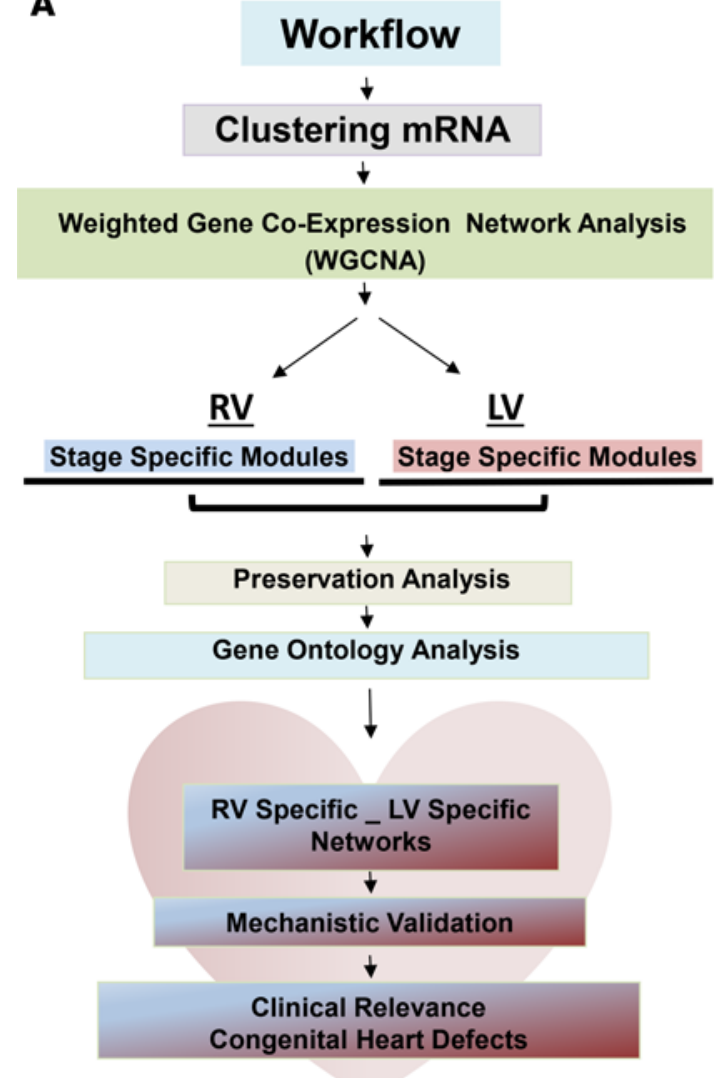

B
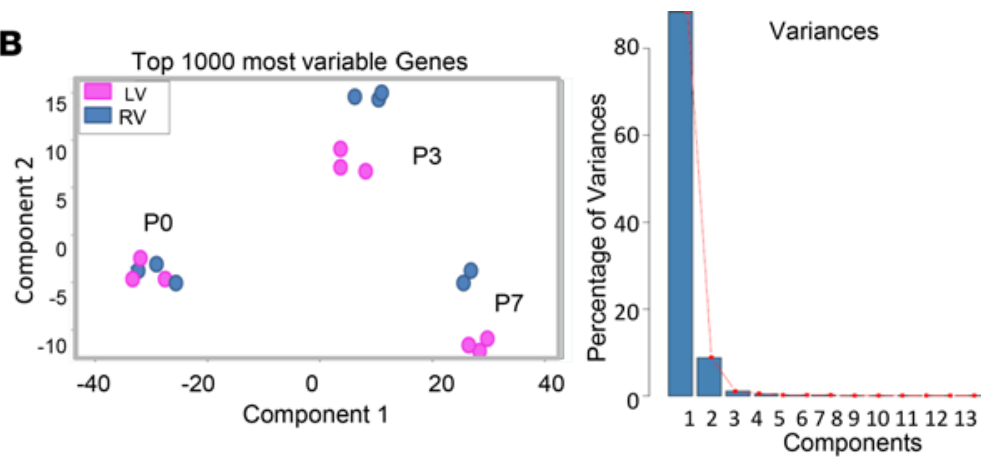

C

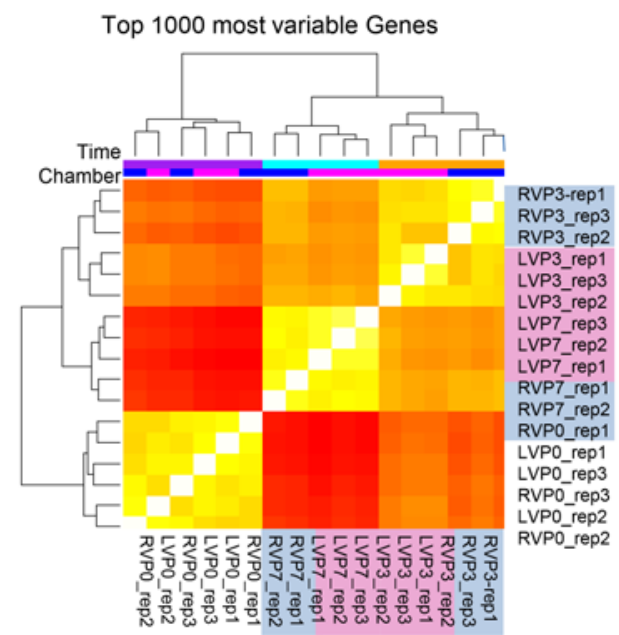

Figure 1. Transcriptome landscape in neonatal mouse heart chambers. (A) Schematic representation of transcriptome analysis workflow. mRNA expression data were derived from deep RNA sequencing data sets of male C57B/6 mouse left ventricles (LVs) and right ventricles (RVs) at postnatal day 0 (PO; before the ductal closure), day 3 (P3; transition), and day 7 (P7; terminal differentiation of the vast majority of CMCs). $n=3$ replicates per chamber per time point, except RVP7 ( $n=2$ replicates). Weighted gene network coexpression analysis (WGCNA) was performed to construct gene coexpression modules in RVs and LVs separately, followed by module preservation analysis and gene ontology analysis to identify chamber-specific genes/gene networks. (B) Principal component analysis (PCA) result of the top 1,000 varied genes (left) and the percentage of variation that corresponds to each component (right). PCA was conducted using R function prcomp. Top 1,000 varied mRNAs based on Tophat alignment results were used to generate PCAs. (C) Unsupervised hierarchical clustering of top 1,000 varied genes derived from 17 RNA-Seq data sets ( $n=3$ replicates per chamber per time point, except RVP7 [ $n=2$ replicates]). Experimental conditions of the samples are demarcated by color bars at the top according to chamber: RV (blue) and LV (pink), and time point: PO (purple), P3 (orange), and P7 (turquoise). Samples from P3 and P7 clustered closely together away from P0, suggesting sharp transcriptome changes during the P0-P3 window. Transcripts from LVs and RVs formed distinct clusters after birth (at P3 and P7), suggesting a temporal chamber-specific transcriptome signature in postnatal heart.

Among the different developmental signals (21-23), Wnt signaling pathways play prominent roles in cardiac precursor's commitment, cell proliferation, differentiation, and fate (24-35).Actions by the Wnt family molecules can be classified into the canonical Wnt/ $\beta$-catenin-dependent pathway and the noncanonical pathway, which is generally $\beta$-catenin independent, and its function can be mediated by 2 distinct modules of the noncanonical signaling: (a) the planar cell polarity (PCP) pathway; and (b) the Wnt/ $\mathrm{Ca}^{2+}$ pathway, which involves calmodulin-dependent kinase II (CamKII), protein kinase C (PKC), and calcineurin. These factors may coordinate important cellular signaling processes including regulating the expression of key cell cycle regulators.

Although substantial overlap exists between the different Wnt signaling pathways during heart development, existing evidence has revealed a critical role of noncanonical Wnt11 in orchestrating early development (24-35). Wnt11 expression is dynamically regulated in a temporal-spatial manner (28), playing important roles in myocardial development, including the second heart field (SHF) derivatives and the outflow tract. Wnt11-null mouse fetuses developed conotruncal defects including pulmonary stenosis and double-outlet RV (DORV) (29). Finally, recent evidence suggests that Wnt11 may also drive the late stages of cardiac myogenesis coupled with inhibition of the canonical Wnt/ $\beta$-catenin (30-32). However, the functional role of Wnt11 in postnatal heart chamber-specific development has not been demonstrated. 
A

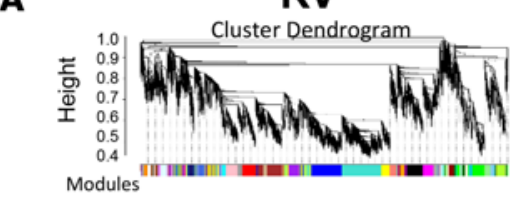

(RPKM $\geq 3, V \geq 0.2$, FDR $P$ Value $\leq 0.05$ )

C

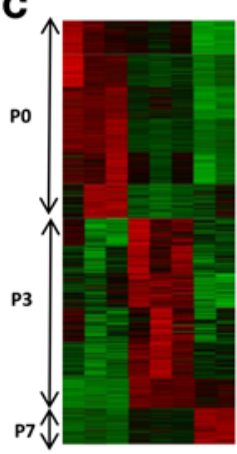

GO Term

Nucleic Acid Metabolism

Cell Cycle

P Value

$3.2 \mathrm{e}-26$

6.3e-18

$1.1 \mathrm{e}-15$

$8.0 \mathrm{e} 24$

$2.2 \mathrm{e}-13$

$1.3 \mathrm{e}-17$

$1.5 \mathrm{e}-09$

6.5e-04

0.007

0.03

0.001

3.1e-11

1.3e-06

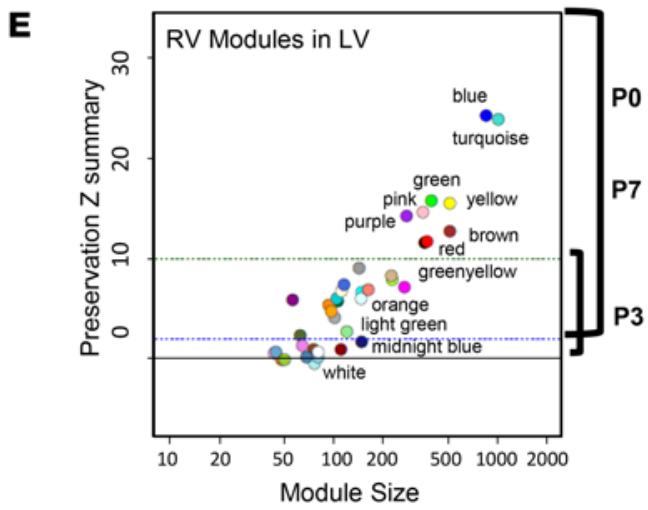

B

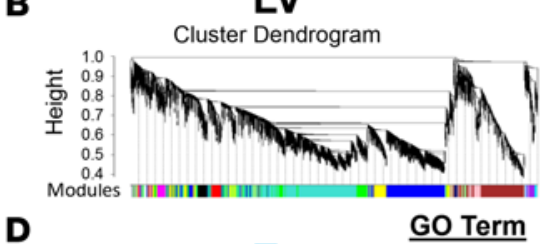

$\underline{P \text { Value }}$

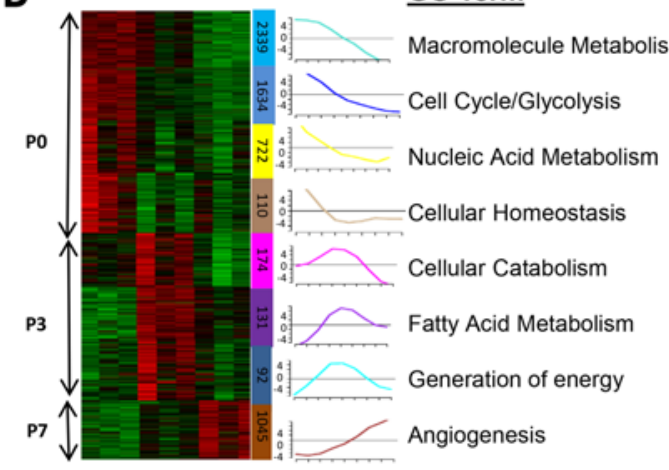

$2.0 \mathrm{e}-42$

3.3e- 07

0.001

0.01

3.1e-19

$2.8 \mathrm{e}-7$

$1.5 \mathrm{e}-10$

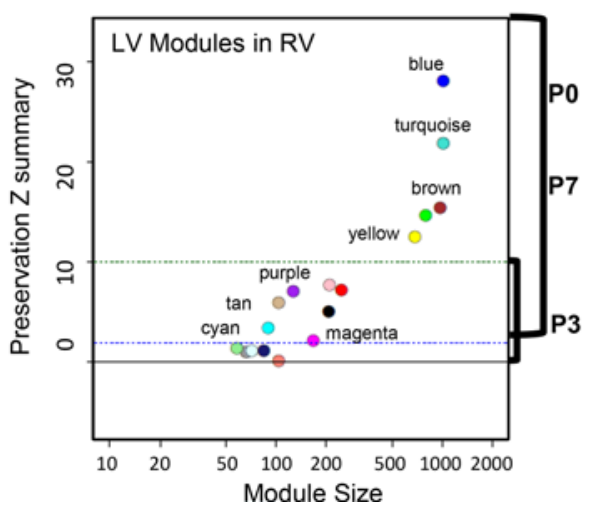

Figure 2. Weighted gene network coexpression analysis (WCCNA) reveals stage-specific modules (gene network) in neonatal left and right ventricles. (A and B) WGCNA dendrograms of right ventricle (RV) (A) and left ventricle (LV) (B) mRNA transcriptome. Genes with mean reads per kilobase of transcript per million mapped reads (RPKM) greater than or equal to 3 in at least one sample (3 replicates) of each category and variation greater than or equal to 0.2 across samples (FDR $P$ value $\leq 0.05$ ) were included from RV and LV data sets separately. Genes are clustered based on the topological overlap, a measure of connection strength. Using the R package, gene modules were constructed as groups of genes with highly similar coexpression relationships. Branches in the hierarchical clustering dendrograms correspond to modules. Color bars below the dendrograms display gene coexpression modules identified by WGCNA in RVs (A) or in LVs (B). The $y$ axes (height) represent module significance (correlation with external trait). (C and D) Heatmaps depict expression profiles of stage-specific mRNA modules' member genes. Thirteen stage-specific modules in RVs (C) and 8 stage-specific modules in LVs (D) are presented with upregulated genes in red, and downregulated genes in green. The expression profiles are standardized; changes are expressed in log2 scale. The numbers of genes corresponding to each module are shown in color bars. Unique stage-specific modules in LVs and RVs are defined as those with Pearson's correlation coefficient $r$ greater than or equal to 0.7 and $P$ value less than or equal to 0.05 between the module eigengene (expression profile summary) and the maturation stage ( $\mathrm{PO}, \mathrm{P3}$, or P7). Eigengene of a given module is presented (bar graphs). Top gene ontology (CO) term enriched in each module along with corresponding adjusted $P$ value is listed to the right of each module. The color code is preserved. (E) Preservation plots of RV modules in LVs, and LV modules in RVs. Module size ( $x$ axis) and preservation Z-summary scores ( $y$ axis) are shown. $Z$ summary less than 2 indicates modules were weakly or not preserved, $Z$ summary between 2 and 10 indicates modules were moderately preserved, and $Z$ summary greater than 10 indicates modules were well or strongly preserved. Color codes of the modules are preserved.

By integrating systems genomic and functional analysis of neonatal LV and RV transcriptomes independently, we established, to the best of our knowledge, the first high-resolution landscape of neonatal chamber-specific transcriptomes. Remarkably, most dramatic changes between LV and RV networks were associated with P3 gene modules, significantly enriched for cell proliferation, DNA damage response, cell cycle regulation, and Wnt signaling. In particular, we identified a previously unrecognized role of Wnt1 in chamber-specific proliferation during the early postnatal window and in response to perinatal hypoxia. Remarkably, the loss of Wnt11 expression is associated with perinatal hypoxia in neonatal mice and in infants with cyanotic $\mathrm{CHD}$ phenotypes. In vivo and in vitro functional studies using targeted loss- and gain-of-function approaches implicate, potentially for the first time, Wnt11 signaling in chamber-specific control of proliferation program under normal conditions and 
during pathological insult by hypoxia. Functional analysis suggested retinoblastoma protein $1(\mathrm{Rb} 1)$ as a potential mediator of Wnt11 function. These insights hold promise for identifying chamber-specific therapeutic targets for early ventricular failure in the infants with cyanotic CHD phenotypes.

\section{Results}

Spatial-temporal transcriptome landscape revealed by longitudinal $R N A$-sequencing analyses. To gain insights into dynamic changes of the cardiac transcriptome during the period of fetal to neonatal transition, we previously carried out systematic analysis of the whole-genome transcriptome of neonatal LV and RV at P0, P3, and P7 derived from deep RNA sequencing (RNA-seq) data sets of the neonatal heart maturation (NHM) SuperSeries (1) (Figure 1A and Methods) and determined that the global signature of cardiac transcriptome was largely dictated by developmental stage rather than chamber identity (Figure 1, B and C, and Supplemental Table 1; supplemental material available online with this article; https://doi.org/10.1172/jci.insight.94904DS1). Specifically, more robust dynamic transcriptome changes were observed during the $\mathrm{P} 0-\mathrm{P} 3$ maturation window in both ventricles, while transcripts from the LVs and RVs formed distinct clusters at P3 and P7, but less so at P0, indicating a progressive establishment of chamber-specific molecular identity in neonatal hearts.

Stage-dependent gene coexpression networks. In an attempt to further analyze the chamber-specific transcriptome regulation of the neonatal heart we reasoned that implementing weighted gene coexpression network analysis (WGCNA) in LV and RV transcriptomes separately would allow us to define modules of genes that are continuously coregulated and to study their stage-specific variation in each ventricle independently along the maturation windows. By including all expressed genes with expression variation greater than or equal to 0.2 in this analysis, we identified a total of $17 \mathrm{LV}$ and $38 \mathrm{RV}$ modules defined as groups of genes coordinately expressed across the $9 \mathrm{LV}$ and $8 \mathrm{RV}$ samples. We used the correlation of the module eigengene, which represents a summary expression profile of the respective module, at the P0, P3, or P7 time point in LVs or in RVs separately to quantify the overall relationship between a module and a trait (maturation stage, chamber). Collectively, we identified $8 \mathrm{LV}$ and $13 \mathrm{RV}$ stage-associated modules, defined as modules that significantly correlated with maturation stage $(r>0.7$ and $P<0.05)$ (Figure 2, A-D). Details of correlation statistics and module sizes are presented in Supplemental Tables 2 and 3. Remarkably, compared with P0 and P7, the magnitude and significance of association between a module and a maturation stage were relatively weaker at P3, indicating larger variability.

Stage-specific modules are preserved between LVs and RVs. To gain further functional insight into global similarities and differences of stage-specific gene modules identified in neonatal heart chambers, we examined whether these modules were preserved between the LV and RV (Figure 2E). Among the developmental stage-associated modules, the P0 modules share significant similarity (as measured by preservation scores) between the LV and RV, and the same pattern applies to the P7 modules. In contrast, the P3 modules exhibited significant variation between the 2 chambers and were either modestly or poorly preserved. Functional enrichment analysis of the preserved time-dependent modules, using the ingenuity pathway analysis (IPA) suite, indicated that the top pathways enriched were also preserved in the LV and RV (Figure 2, C and D).

Intriguingly, among the P3-associated modules, the LV Purple and its counterpart the RV Light Green module turned on robustly during the P0-P3 transition to become the most correlated with the P3 time point. Genes from these modules were preserved between the 2 ventricles and enriched in fatty acid metabolism and PPAR signaling, indicating concordant activation of the fatty acid metabolism pathway at P3. Using quantitative reverse transcription PCR (qRT-PCR) we demonstrated that the expression levels of 14 selected genes involved in fatty acid $\beta$ oxidation and glycolysis exhibited reciprocal regulation between the $\mathrm{P} 0$ and $\mathrm{P} 3$ time points, indicating a synchronized molecular switch from glucose metabolism to fatty acid utilization pathway, as has been previously reported $(36,37)$. This suggests that metabolic switch occurs as early as P3 in both ventricles in the neonatal mouse heart (Supplemental Figure 1).

Chamber-specific gene networks involving Wnt signaling and cell cycle genes. Noting that $\mathrm{P} 3$-associated modules are the least preserved between the LVs and the RVs, we characterized the poorly preserved P3 modules in more detail (Figure 3A). In total, 117 cell cycle-related genes exhibited stage- and chamber-dependent downregulation. For example, genes involved in the polo-like kinase mitotic entry pathway (PLK1, CCNB1, CCNB2, and Cdc25c) were rapidly suppressed in the RV during the P0-P3 maturation window. This transition was slower in the LV, predominantly during the P3-P7 window (Supplemental Figure 2). Likewise, the ATM/DNA damage response CHK1 pathway displayed different temporal regulation between LVs and RVs. Together, the differential expression of cell cycle progression and control genes at P3 
A

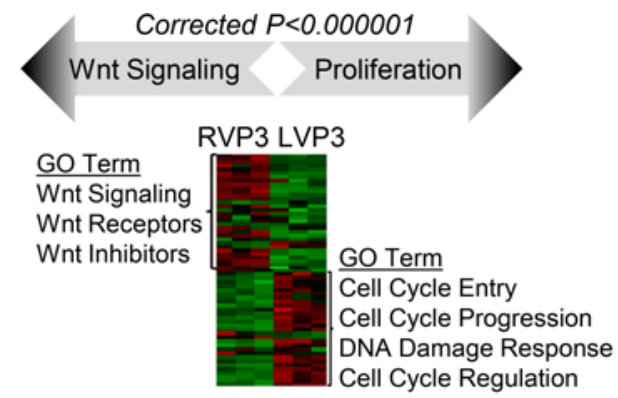

B
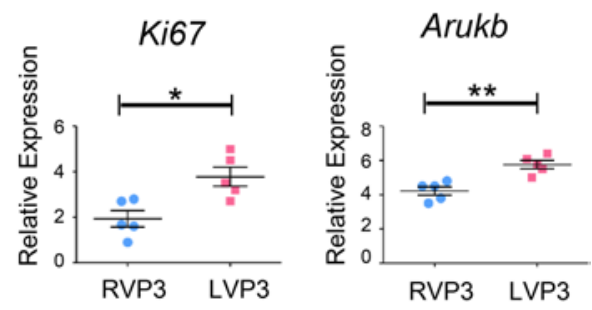

C Tropomyosin DAPI $\mathrm{pH} 3$
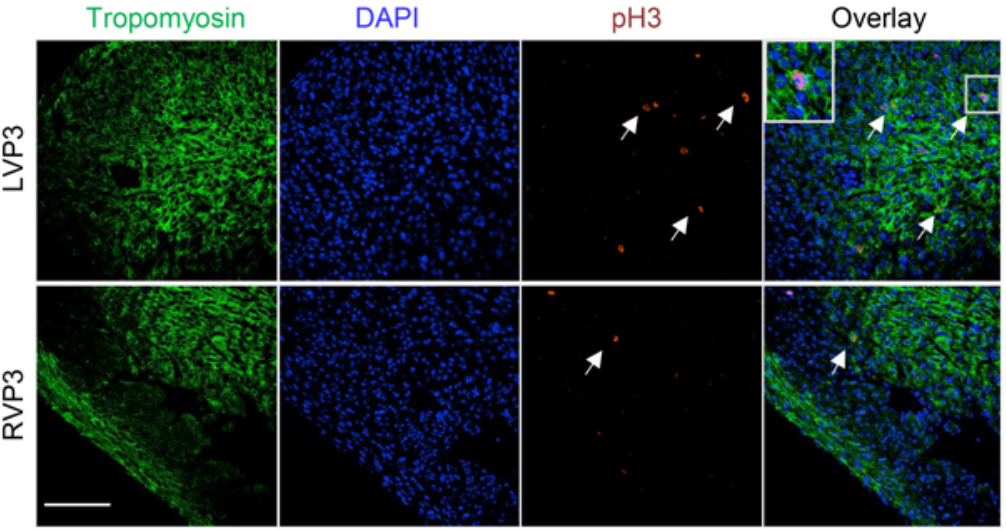

D
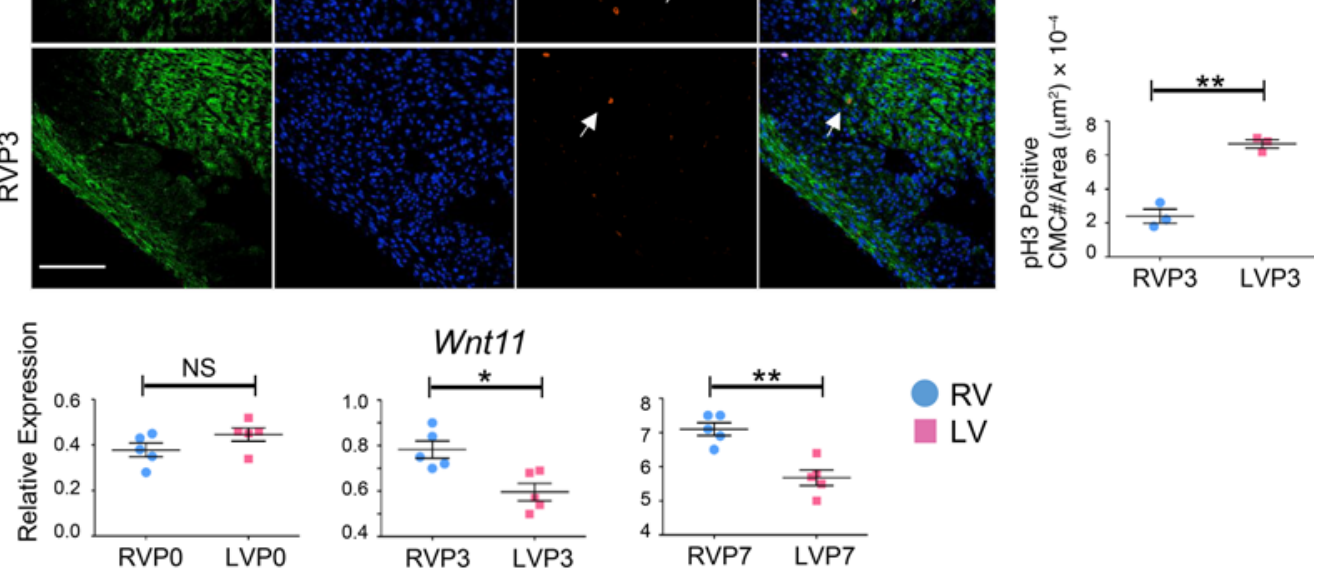

E
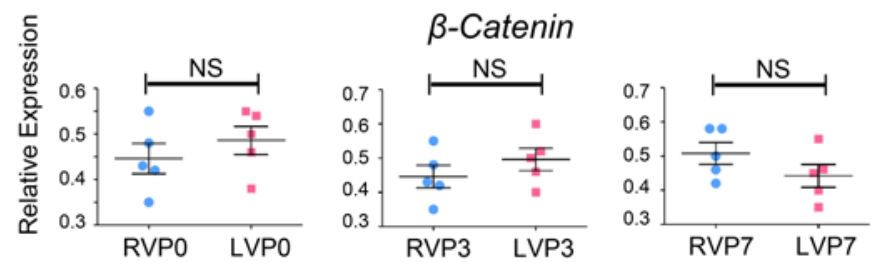

$\mathbf{F}$
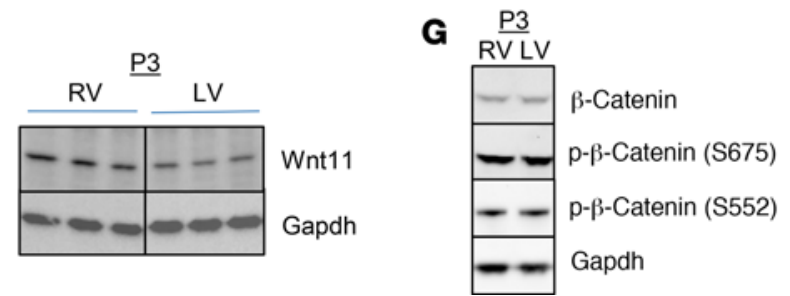

Figure 3. Chamber-specific enrichment of cellular proliferation and Wnt signaling revealed by neonatal heart transcriptome analysis. (A) Gene ontology (GO) analysis depicting chamber-specific enrichment of Wnt signaling and cell cycle processes in P3 poorly preserved modules. Expression heatmap of cell proliferation/cell cycle genes (enriched in left ventricular [LV] modules) and Wnt signaling genes (enriched in right ventricular [RV] modules) in P3 neonatal heart is shown. Expression changes are expressed in log2 scale. Red, upregulated; green, downregulated. (B) qRT-PCR analysis of proliferation marker Ki67 and mitosis marker aurora kinase B (AurkB) in LV or RV myocardium of WT neonatal mouse $(n=5)$ at P3. Blue, RV; pink, LV. Error bars represent SEM. (C) Representative confocal images of anti-phospho-histone H3 (anti-p-H3) immunohistochemistry (IHC) in RVs or LVs of WT neonatal mouse heart at P3. Arrows indicate p-H3-positive cardiomyocytes (CMCs). Original magnification, $\times 40$. (D and E) qRT-PCR analysis of Wnt11 and $\beta$-catenin in LV or RV myocardium of WT neonatal mouse, PO through P7 $(n=5)$. Blue, RV; pink, LV. Error bars represent SEM. (B-E) by unpaired, 2-tailed Student's $t$ test. Data are representative of 3 independent experiments. (F) Wnt11 protein expression in LV and RV myocardium of WT neonatal mouse hearts at P3 (Western blot). Gapdh was used as loading control. $n=3$ replicates per condition. See related Supplemental Figure 3. (C) Expression of $\beta$-catenin protein and its phosphorylated forms at Ser675 and Ser552 (S675 and S552) in LV and RV myocardium of WT neonatal mouse hearts at P3 (Western blot). Gapdh was used as loading control. $n=3$ replicates per condition. NS, not significant. ${ }^{*} P \leq 0.05$, ${ }^{* *} P \leq 0.01$. 
A O2 Sat
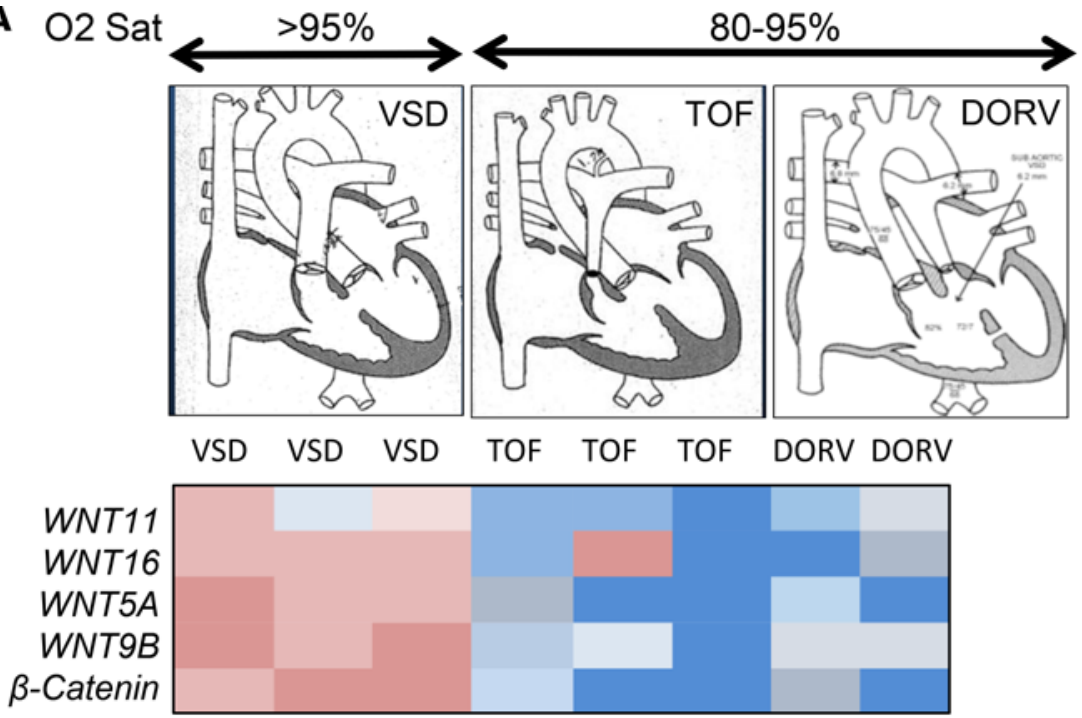

B

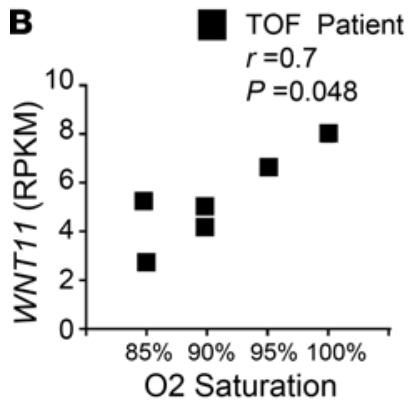

Human RNA-Seq Data

TOF Patient
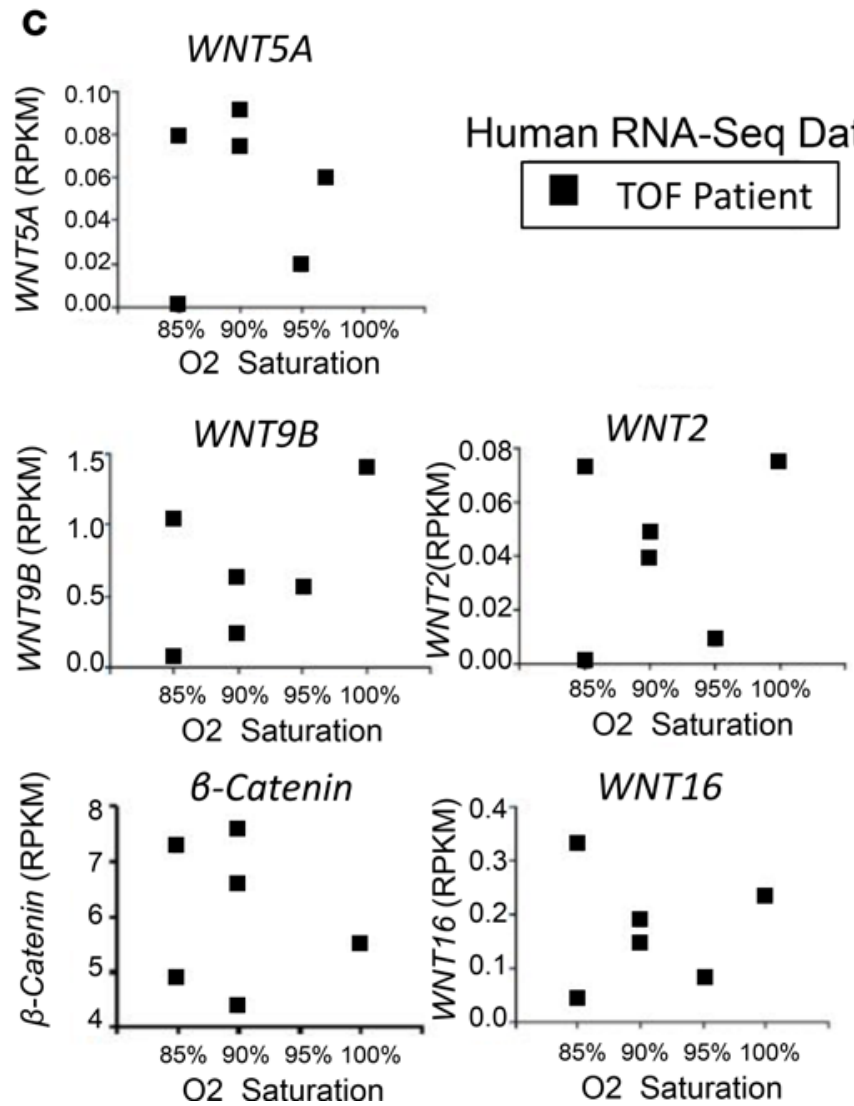

Figure 4. Wnt11 may play important roles in human congenital heart defects (CHDs).

(A) Expression array of Wnt molecules and cell cycle genes in cyanotic (hypoxemic) versus noncyanotic (nonhypoxemic) human CHD phenotypes. Graphs: Representative illustrations of CHD cases. VSD, ventricular septal defect $(n=3)$; TOF, Tetralogy of Fallot $(n=3)$; DORV, double-outlet right ventricle $(n=2)$. Red, upregulated; blue, downregulated. (B) Correlation plot demonstrating Wnt11 association with preoperative $\mathrm{O}_{2}$ saturation levels in heart tissue specimens from TOF patients with different baseline $\mathrm{O}_{2}$ saturation levels $(n=6)$. $r$, Pearson's correlation coefficient; $P$, correlation $P$ value. Wht11 expression data were obtained from $\mathrm{M}$. Touma's unpublished observations (RNA-Seq data set) of human heart tissue of patients with TOF. RPKM, reads per kilobase of transcript per million mapped reads. (C) Correlation plots revealed no association between the expression of several WNT genes (WNT5A, WNT9B, WNT2, WNT16, and $\beta$-catenin) and preoperative $\mathrm{O}_{2}$ saturation levels in a new set of TOF patients ( $n$ $=6$ ) with different baseline $O_{2}$ saturation levels. The expression data (RPKM) were obtained from RNA-Seq of heart tissue of patients with TOF (M. Touma's unpublished observations [RNASeq data set]).

modules revealed a temporary lag in cell cycle regulation in LVs compared with RVs during perinatal transition. Consistent with these observations, the proliferation markers phospho-histone H3-positive (p-H3positive) CMCs and Ki67 were increased, and the mitotic marker Aurkb (aurora kinase B) was significantly induced in LVs compared with RVs in wild-type neonatal mice at P3 (Figure 3, B and C).

Among the major pathways enriched in the P3 poorly preserved modules, we found that 27 out of 58 Wnt-related genes were regulated in a chamber-specific manner, including components from the canonical and noncanonical signaling pathways (24-35). Remarkably, established inhibitors of canonical $\beta$-catenindependent signaling (Wif1, Dkk3, and Sost1) were upregulated in RVs compared with LVs, while several members of the noncanonical Wnt11 signaling (Wnt11, Wnt5a, Ror2, Sfrp5, and Rock2) were enriched 
in RVs. Consistently, qRT-PCR and Western blot revealed RV-enriched expression of the noncanonical Wnt1 in P3 neonatal mouse heart (Figure 3, D and F). In contrast, $\beta$-catenin and $p$ - $\beta$-catenin, as important markers for the canonical Wnt/ $\beta$-catenin-dependent signaling activity, did not exhibit significant chamber-specific regulation in neonatal heart (Figure 3, E and G). Together, our observations suggest a prominent chamber-specific enrichment of Wnt1 that is reciprocally correlated with cellular proliferation in a chamber-specific and developmentally regulated manner.

Wnt signaling in human congenital heart defects. To explore the clinical relevance, specimens from the RV outflow tract (RVOT) were obtained from 3 infants with ventricular septal defect (VSD), 3 infants with Tetralogy of Fallot (TOF), and 2 infants with DORV (Figure 4A). Infants with TOF or DORV presented with cyanosis and were hypoxemic $\left(\mathrm{O}_{2}\right.$ saturation $\left.<85 \%\right)$. Intriguingly, the altered expression of up- or downregulated Wnt signaling molecules exhibited distinct patterns in cyanotic (TOF, DORV) versus noncyanotic (VSD) patients. Next, we performed RNA-Seq on a new group of infants ( $<1$ year old) with isolated, nonsyndromic TOF who presented with different degrees of cyanosis and hypoxemia (M. Touma's unpublished observations). Importantly, among the different Wnt ligands that were expressed in infantile TOF heart (Figure 4, B and C), Wnt11 exhibited the most significant correlation with average preoperative $\mathrm{O}_{2}$ saturation levels (lower Wnt11 expression was associated with lower $\mathrm{O}_{2}$ saturation) in the TOF infants, raising questions about a Wnt11 signaling role in the RV-specific impact of hypoxia in postnatal infantile heart with cyanotic CHDs.

Perinatal hypoxia induced CMC proliferation and suppressed Wnt11 in a chamber-specific manner. Hypoxia is a major stress factor during fetal to neonatal transition, especially in the context of a CHD. To test the impact of hypoxia on neonatal heart chamber proliferation we exposed newborn mouse pups to systemic hypoxia (fraction inhaled $\mathrm{O}_{2}\left[\mathrm{FIO}_{2}\right] 10 \%$ ) during perinatal transition (Figure 5A). Perinatal hypoxia $\left(\mathrm{FIO}_{2}\right.$ $10 \%$ ) suppressed Wnt11 RNA and protein expression more robustly in RVs compared with LVs (Figure 5, B and C, and Supplemental Figure 3), while more significantly induced CMC proliferation was observed in RVs (Figure 5, D-F). Remarkably, hypoxia-exposed pups developed more robust induction of cellular proliferation in RVs compared with LVs, as demonstrated by the enhanced phosphorylation of histone $\mathrm{H} 3$ protein, reaching levels comparable to that observed in LVs at P3 (Figure 5D).

Perinatal hypoxia modulates $R b 1$ activity in a chamber-specific manner. Among several cell cycle regulatory genes and Wnt11 signaling mediators that we screened (Figure 5, G and $\mathrm{H}$ ), Rb1, a key negative regulator of cell cycle entry (38-40), was found to be temporally regulated and differentially altered in RVs compared with LVs in response to hypoxia treatment (data not shown). Rb1 was lower in protein abundance in RVs versus LVs throughout P0 to P7. However, total Rb1 protein increased substantially following hypoxia in both ventricles at P0 and P3, leading to diminished differences between RVs and LVs. Remarkably, enhanced p-Rb1 at Ser807 (S807), S811, and S780 sites was observed in both ventricles in response to hypoxia treatment. Specifically, p-Rb1 at S807 and S811 in RVs reached levels similar to those observed in LVs at P3 (Figure 5H). Collectively, these data suggest that perinatal hypoxia modulates Rb1 activity in a chamber-specific manner, predominantly affecting RVs, at mRNA, protein, and posttranslational modification levels, leading to decreased $\mathrm{Rb} 1$ activity and enhanced cell proliferation.

Chamber-specific roles of Wnt11 in cardiac proliferation. To assess Wnt11 function in neonatal myocardium growth, we performed Wnt11-specific inhibition via Wnt11-Vivo-Morpholino injection into pregnant mice at late gestation (Figure 6A), which did not immediately affect cardiac function (Supplemental Figure 4). As shown in Figure 6B, the number of p-H3-positive CMCs was increased following Wnt11 inhibition to reach similar numbers in both ventricles. However, the p-H3-positive $\mathrm{CMC}$ increment was more robust in RVs. Likewise, the proliferation markers were induced significantly in RVs, and trended up in LVs (Figure 6C), including mitosis genes AurkB, Plk1, and their mitotic substrates, Cdc25c and ECT2. Mitotic spindle assembly-marker genes Bub1 and Cenpf were also induced more robustly in RVs compared with LVs. Collectively, these data suggest that noncanonical Wnt11 regulates neonatal heart proliferation in a chamber-specific and temporally regulated manner.

Wnt11 regulates neonatal CMC proliferation. Wnt11's role in postnatal chamber growth may involve any of the different cell types that reside in the growing chambers. To determine the cell-autonomous function of Wnt11 in neonatal CMCs specifically, suppression of Wnt11 was achieved in neonatal rat ventricular myocytes (NRVMs) in culture (Figure 7, A and B). The cells exhibited elongated shape with increased p-H3-positive CMC number and induced Ki67 expression (Figure 7, A, C, and D). In contrast, the myocytes remained quiescent in response to exogenous recombinant Wnt11 (rWnt11) and the binucleation index was increased in rWnt11-treated cells, measured after excluding all p-H3-positive CMCs in this 
A

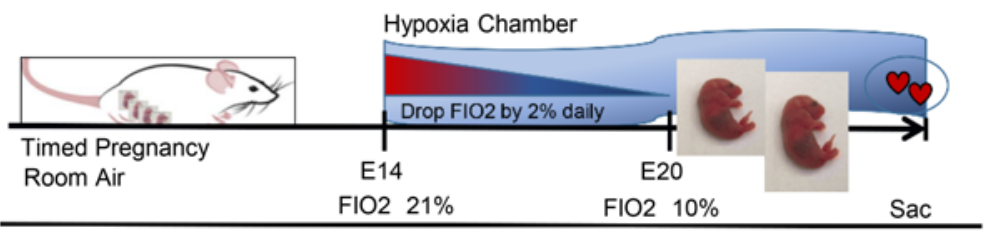

B

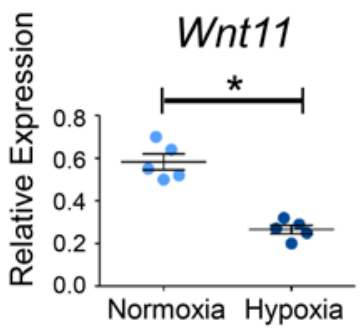

RV/Normoxia

RV/Hypoxia

- LV/Normoxia

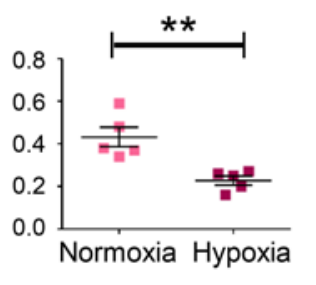

C

Normoxia Hypoxia Normoxia Hypoxia

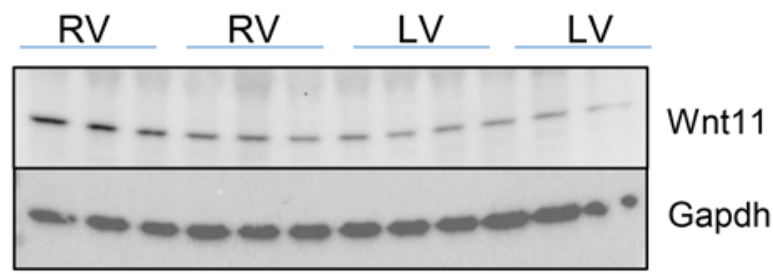

D

Normoxia

Hypoxia

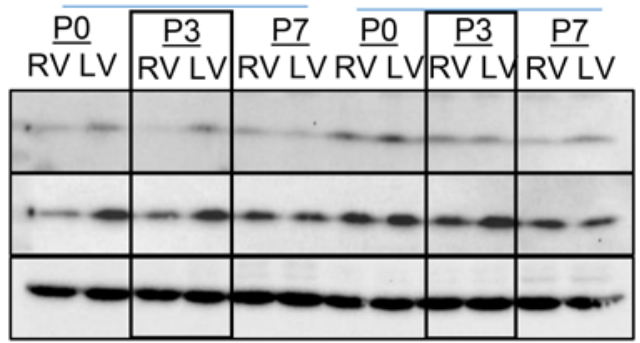

$\mathrm{H} 3$

$\mathrm{pH} 3(\mathrm{~s} 10)$

$\beta$-Actin

G

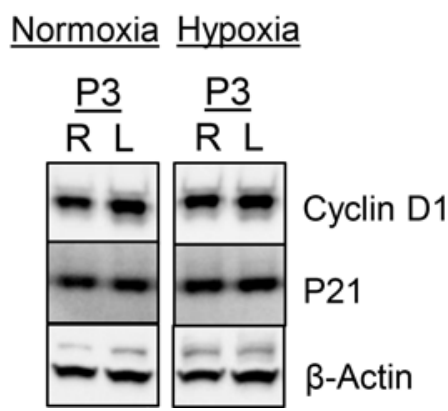

H
$\mathbf{E}$

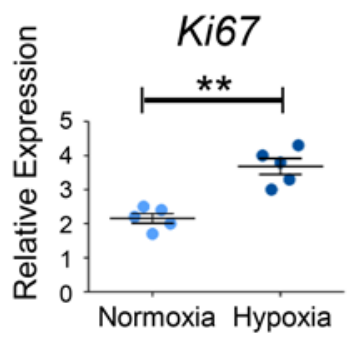

F

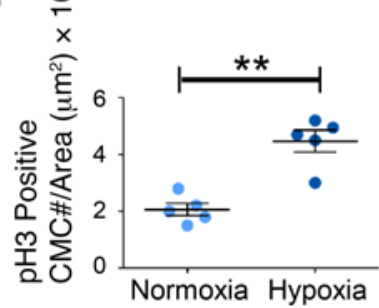

Ki67
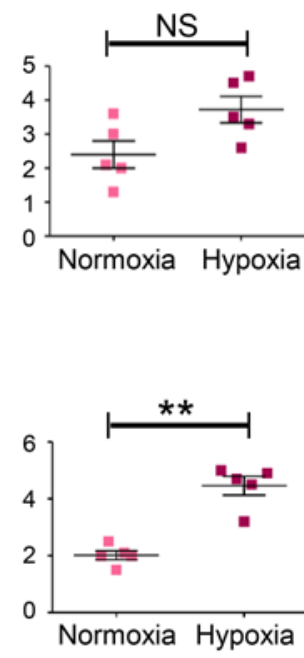

\section{LV/Hypoxia}
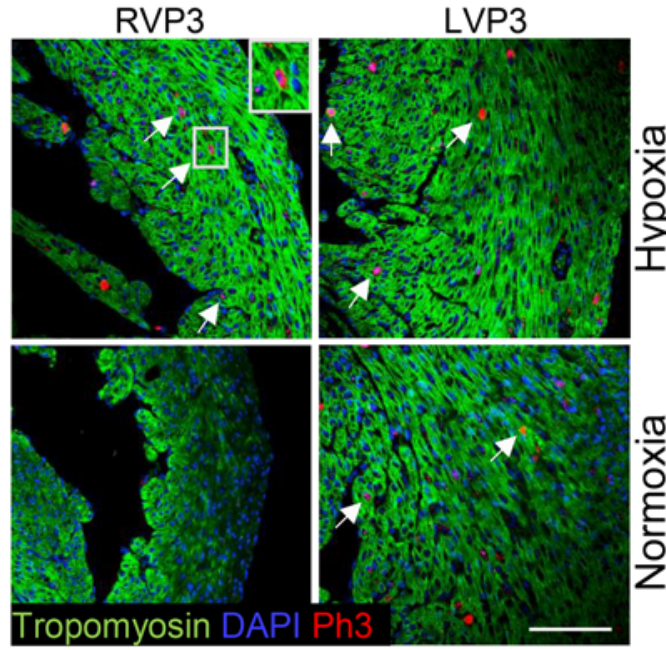

Normoxia

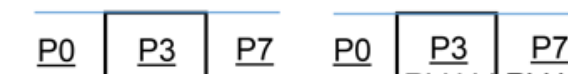
RV LV RV LV RV LVRV LVRVLVRV LV

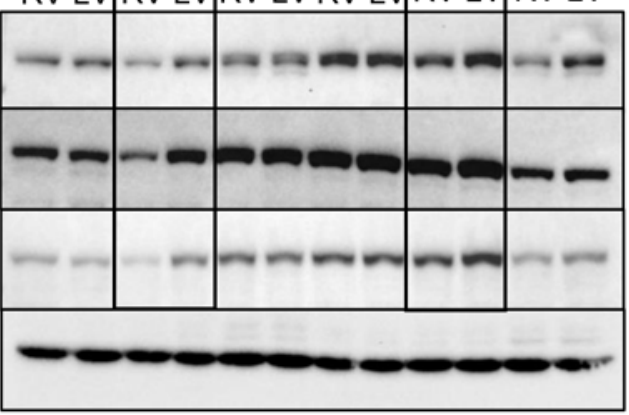

Total Rb1

$\mathrm{p}-\mathrm{Rb} 1$ (S807/811)

p-Rb1

$(\mathrm{S} 780)$

$\beta$-Actin

Figure 5. Hypoxia regulates neonatal cardiomyocyte proliferation in a chamber-specific manner. (A) Schematic illustration of experimental design for perinatal systemic hypoxia exposure. Neonatal pups were reared with their dams in either normoxia or hypoxia $\left(10 \% \mathrm{FIO}_{2}\right)$ and maintained until the predecided end points ( $\mathrm{PO}, \mathrm{P} 3$, or $\mathrm{P} 7)$. The dams carrying the experimental group (hypoxia) were acclimatized in the hypoxia chamber by decreasing $\mathrm{FIO}$, by $2 \%$ daily for at least 5 days preceding the experiment starting at E14.5 to reach 10\% on E19.5. (B) Wnt11 mRNA expression in left ventricular (LV) and right ventricular (RV) myocardium of WT neonatal mouse at P3 in normoxic and hypoxic conditions (qRT-PCR). $n=5$ replicates per condition. (C) Wnt11 protein expression in LV 
and RV myocardium of WT neonatal mouse at P3 in normoxic and hypoxic conditions (Western blot). Gapdh was used as loading control, $n=3$ replicates per condition. See related Supplemental Figure 3. (D) Expression time course of histone H3 protein and p-H3 at Ser10 (S10), P0 through P7, in LV or RV myocardium of WT neonatal mouse hearts in normoxic or hypoxic condition (left panel). $n=5$ replicates per condition. (E) Expression of proliferation marker Ki67 for LV or RV myocardium of WT neonatal mouse hearts in normoxic or hypoxic conditions (qRT-PCR). $n=5$ replicates per condition. (F) Representative confocal images of anti-phospho-histone H3 (anti-p-H3) immunohistochemistry (IHC) in LVs and RVs of WT neonatal mouse heart at P3 in normoxic and hypoxic conditions. Arrows: representative $\mathrm{p}-\mathrm{H} 3-$ positive cardiomyocytes. Original magnification $\times 60$. Graph: quantitative analysis of $\mathrm{p}-\mathrm{H} 3-\mathrm{positive} \mathrm{cells} \mathrm{(cell} \mathrm{number/area} \mathrm{[} \mu$ m2], $n=5$ replicates per condition. (G) Expression of cyclin D1 and P21 proteins in LV or RV myocardium of P3 WT neonatal mouse hearts in normoxic or hypoxic condition. $\beta$-Actin was used as loading control. $n=5$ replicates per condition. (H) Expression time course of Rb1 protein and phosphorylated Rb1 ( $p$-Rb1) at Ser807/811 and Ser780 (S807/811 and S780), P0 through P7, in LV or RV myocardium of WT neonatal mouse hearts in normoxic or hypoxic condition ( $n=5$ replicates per condition). Error bars represent SEM. ${ }^{*} P \leq 0.05,{ }^{*} P \leq 0.01$ by unpaired, 2-tailed Student's $t$ test.

analysis in order to determine the accumulated effect of karyokinesis without following cytokinesis as a hallmark of neonatal CMC maturation (Figure 7, A and E). No significant changes were observed in Ki67 expression following rWnt11 treatment (data not shown); however, cyclin D1 was suppressed (Figure 7F).

Together, these data indicate that Wnt11 regulates proliferation programs of neonatal CMCs. Remarkably, loss of endogenous Wnt11 was associated with suppressed total Rb1 protein expression and phosphorylation without impacting JNK and PKC $\alpha$ expression or phosphorylation (Figure 7, G and $\mathrm{H}$ ), suggesting that $\mathrm{Rb} 1$ is a possible target for Wnt11, potentially mediating Wnt11 roles in neonatal CMC proliferation.

Wnt11 loss in cyanotic TOF infants is associated with reduced Rb1 expression and induction of a mitotic marker. Intriguingly, our human studies suggested significant correlation between hypoxia degree, or oxygen saturation level, and Wnt11 expression level in human TOF patients, raising the question whether Wnt11 suppression in these hypoxemic infants can contribute to cell cycle regulation and potential regenerative activity in their hearts. Using qRT-PCR we validated reduced Wnt11 expression in a new group of cyanotic (hypoxemic) TOF infants compared with TOF infants with normal oxygen saturation level. Next, we examined the expression of $\mathrm{Rb} 1$ and proliferation marker genes. Remarkably, Wnt11 loss was associated with $\mathrm{Rb} 1$ reduction. In contrast, the mitotic marker gene Plk1 was enhanced in cyanotic TOF patients, inversely associating with both Wnt11 and Rb1 (Figure 8A). Furthermore, p-H3-positive CMCs were enhanced in heart specimens from cyanotic TOF infants (Figure 8B).

\section{Discussion}

In this study, we performed genome-wide transcriptome analysis within specific cardiac chambers (LV vs. RV) at perinatal maturation time points. We identified $13 \mathrm{LV}$ and $8 \mathrm{RV}$ coexpression gene modules that were significantly associated with maturation stages and enriched with several cellular and signaling processes. In particular, we found that Wnt11 displayed chamber-specific expression patterns. Loss- and gain- of-function studies in vitro and in vivo demonstrated a direct impact of Wnt11 on myocardial proliferation. More importantly, Wnt11 expression was modulated by hypoxic stress in neonatal hearts and its expression was highly associated with hypoxemic (cyanotic) conditions in the CHD infantile hearts. Therefore, we utilized a systems biology approach followed by functional validation analysis to elucidate a, to our knowledge, novel Wnt11-mediated molecular network that governs cardiac chamber-specific growth during the perinatal maturation window, which may contribute to hypoxia-induced pathological maturation of heart chambers among CHD patients.

LV and RV chambers undergo dramatically different growth patterns in postnatal stages. However, while several previous analyses focused on neonatal heart transcriptome changes during the postnatal period $(36,37)$, chamber-specific transcriptome profiling during perinatal (fetal to neonatal) transition is currently lacking. To our knowledge, our current study provides the first analysis of neonatal heart ventricle-specific transcriptome during perinatal circulatory transition at a high level of resolution. Our comprehensive analysis reveals that a developmentally driven core transcriptional program is shared in both LVs and RVs despite the differences in morphological, hemodynamic, and functional characteristics. Nevertheless, some of the key gene expression programs, such as cell proliferation, diverge in their temporal specificity between LVs and RVs, showing chamber-specific gene induction or suppression waves in critical development windows. This is particularly obvious for P3, which appears to be an auxiliary stage when RV and LV time-associated modules either converge, reflecting a developmental program, or diverge, reflecting chamber specificity and adaptation to hemodynamic and environmental changes. These insights may implicate a chamber-specific regenerative window and proliferative capacity, or outcome to stress at a given postnatal time point. 
A
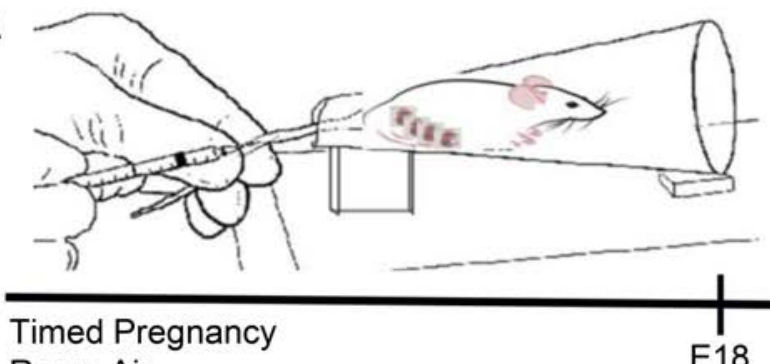

Room Air

B
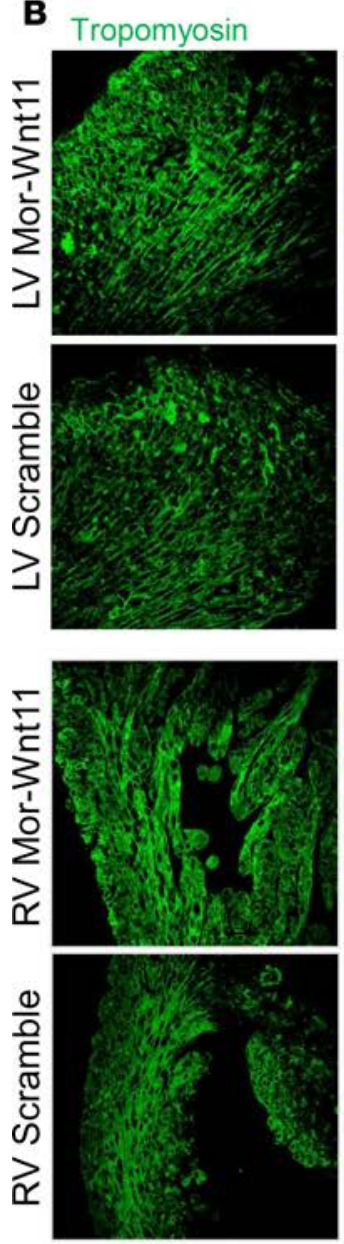

C
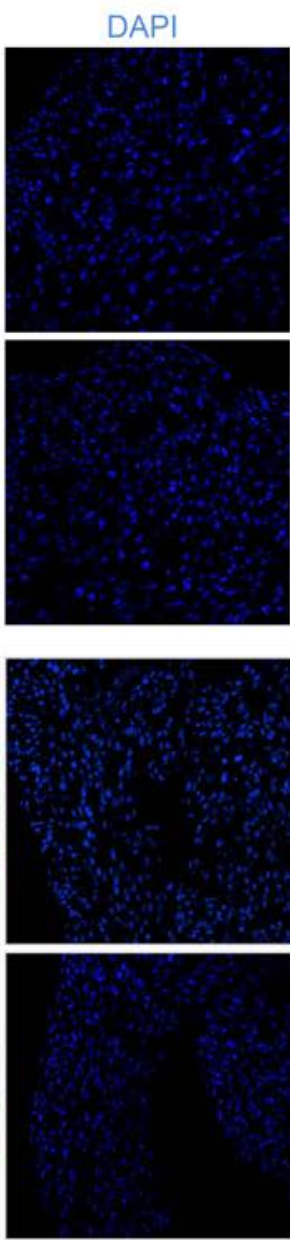
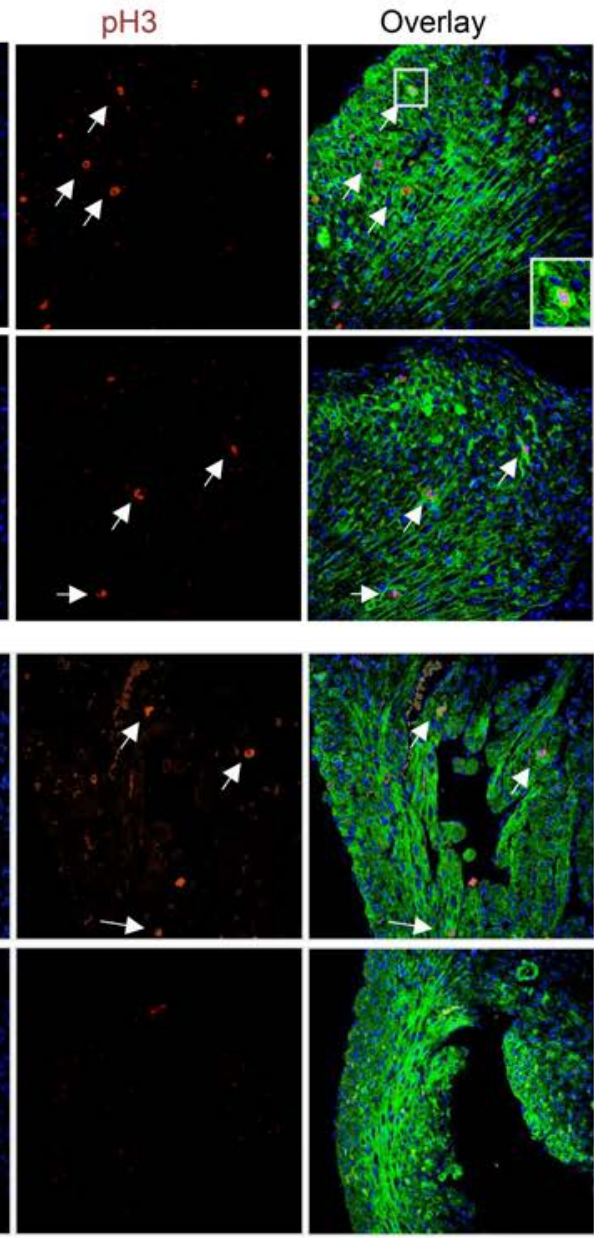

Scramble Mor-Wnt11

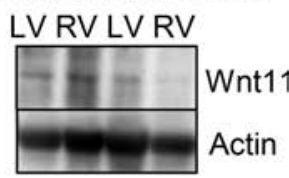

Inhibition Efficiency
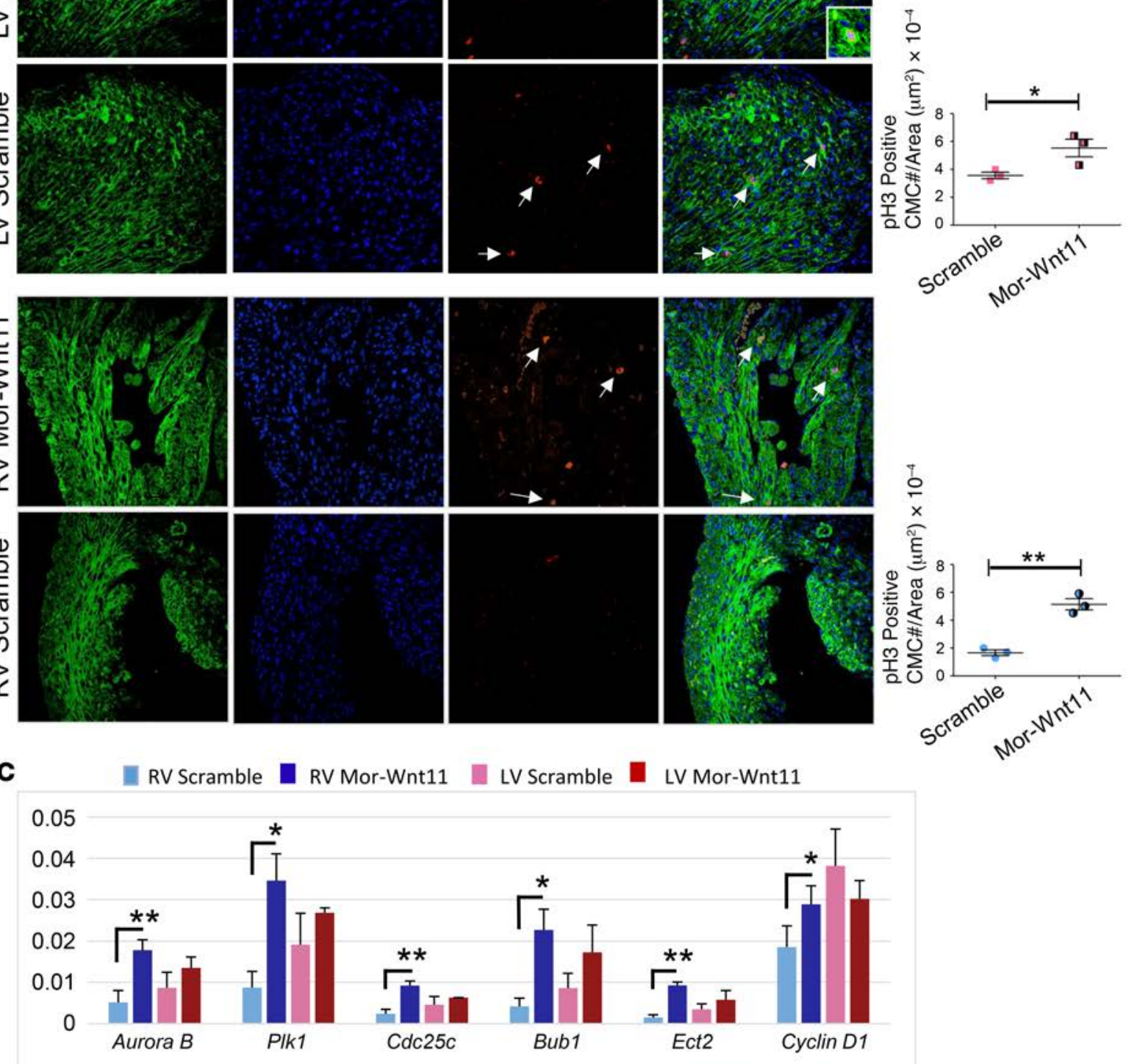

Figure 6. Systemic Wnt11 inhibition induces cardiomyocyte proliferation in a chamber-specific manner. (A) Schematic illustration of experimental design for in vivo Wnt11 knockdown. Analysis of inhibition efficiency (Western) is shown (right panel). Mor-Wnt11 (Vivo-Morpholino-Wnt11): Wnt11-specific modified antisense oligonucleotide. (B) Representative confocal images of anti-phospho-histone $\mathrm{H3}$ (anti-p-H3) immunohistochemistry (IHC) in scramble control or Mor-Wnt11-injected neonatal mouse hearts at P3. Arrows indicate representative p-H3-positive cardiomyocytes (CMCs). Original magnification, 
$\times 40$. Graphs: Quantitative analysis of p-H3-positive cells (cell number/area [ $\left.\mu \mathrm{m}^{2}\right]$. (C) Expression analysis of several proliferation and mitosis markers using mRNA from LV or RV myocardium of Mor-Wnt11- or scramble-treated neonatal mouse (qRT-PCR). $n=3$ per condition. Data are representative of 2 independent experiments. Error bars represent SEM. ${ }^{*} P \leq 0.05,{ }^{* *} P \leq 0.01$ by unpaired, 2-tailed Student's $t$ test (Mor-Wnt11 compared with control).

The noncanonical Wnt11 is a relatively new and underexplored member of the Wnt family, and its role in postnatal heart maturation or disease has not been reported. It was previously shown that Wnt11 is required for proper cardiogenesis, myocardium development, and outflow tract morphogenesis (27-29), while a recent study also implicated Wnt11 in reducing heart scaring after infarction (41). Our current report proves, to our knowledge for the first time, that endogenous Wnt11-mediated signaling directly regulates neonatal CMC proliferation. Wnt11 expression is suppressed by moderate hypoxia and is associated with enhanced CMC proliferation in a chamber-specific manner. Furthermore, a global perinatal inhibition of Wnt11 using Vivo-Morpholino knockdown is sufficient to induce myocyte proliferation in a chamber-specific manner. To our interest, despite systemic and global Wnt11 knockdown, we observed induction of proliferation markers predominantly in RVs. Therefore, the data collectively suggest that Wnt11-mediated signaling is a promising pivotal modulator of chamber-specific myocardium growth and adaptation to postnatal hypoxia. However, there are clear limitations in specificity and efficiency of the Vivo-Morpholino approach. Future rigorous analysis using an inducible CMC-specific Wnt11-deficient mouse model will be needed to fully establish the chamber-specific role of Wnt1 in neonatal CMC proliferation in vivo.

Our findings raise the question of whether relatively temporal and subtle changes in Wnt11 are sufficient to evoke significant changes at the cellular level. Prior studies have demonstrated critical roles of the temporal regulation of Wnt1 in cardiac lineage commitment and differentiation (25, 26). In addition, Wnt1 has been implicated as an important modulator for neuronal maturation and identity $(42,43)$. However, the downstream signaling mechanism for Wnt11-mediated regulation of myocyte proliferation needs to be further investigated. Our data neither support a chamber-specific distribution of $\beta$-catenin (as a surrogate marker of the canonical Wnt signaling activity) nor reveal a chamber-specific degradation of $\beta$-catenin via phosphorylation (Figure $3, \mathrm{~F}$ and $\mathrm{H}$ ). In contrast to $\beta$-catenin and other known players in cellular proliferation (P21 and cyclin D1), Rb1 was clearly implicated in Wnt11-mediated regulation of CMC proliferation in our study. Rb1, a well-established suppressor of cell cycle entry, has been implicated in controlling terminal differentiation in many tissues, including the heart (38-40). Importantly, Rb1 is known to be regulated by hypoxia in cancer cells (44). Here we demonstrated that hypoxia modulates Rb1 activity, leading to chamber-specific induction of cell cycle genes in neonatal heart. Our study establishes that Wnt11 modulates Rb1 expression and activity, and potentially mediates hypoxia-induced proliferation.

How Wnt11 signaling regulates Rb1 activity and neonatal CMC proliferation in neonatal heart chambers remains to be fully investigated. Our preliminary screening of known Wnt11 mediators, JNK, PKC $\alpha$, and p38, did not support their direct involvement (Figure 7G). However, it is well known that Wnt11 function may be mediated via $\mathrm{Ca}^{2+}$ signaling $(45,46)$, which, in turn, coordinates cellular proliferation genes via regulating the $\mathrm{Rb} 1 / \mathrm{E} 2 \mathrm{~F} 1$ pathway. In its hypophosphorylated state $\mathrm{Rb} 1$ binds to $\mathrm{E} 2 \mathrm{~F} 1$ and represses the expression of $\mathrm{E} 2 \mathrm{~F} 1$ target genes. In doing so, Rb1 restricts the expression of cell cycle entry genes. In contrast, phosphorylation of Rb1 leads to release of the E2F1 transcription factor which, in turn, activates G1-S transition and promotes cell cycle progression. The coordination of these events is critical for appropriate regulation of cell cycle leading to mitosis in cancer. Importantly, calcineurin, an established intercellular mediator of $\mathrm{Wnt} 11 / \mathrm{Ca}^{2+}$ signaling, may dephosphorylate $\mathrm{Rb} 1$ at $\mathrm{S} 795$, leading to its interaction with E2f1, recruitment of Hdac1, and suppression of cell cycle entry, as demonstrated previously in neuronal cells $(42,43)$. It would be interesting to determine whether calcineurin could dephosphorylate $\mathrm{Rb} 1$ at $\mathrm{S} 795$, leading to $\mathrm{Rb} 1$ activation and enhanced $\mathrm{Rb} 1 / \mathrm{E} 2 \mathrm{f1}$ interaction, thereby restricting cell cycle entry in neonatal myocardium.

Perinatal hypoxia induces myocyte proliferation more robustly in RVs and suppresses Wnt11 expression in a chamber-specific manner, eliminating the differences in myocyte proliferation between LVs and RVs observed under normal conditions. More importantly, we discovered, to our knowledge for the first time, differential activation of Wnt pathways in infants with cyanotic (hypoxemic) versus noncyanotic (nonhypoxemic) CHDs (Figures 4 and 8). Specifically, Wnt11 significantly correlate with $\mathrm{O}_{2}$ saturation in TOF phenotypes. Previous studies $(19,20)$ have demonstrated that hypoxic (low oxygen tension) microenvironment, or niches, may play a 
A

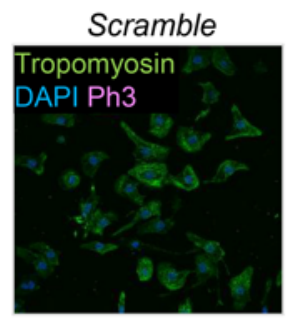

B
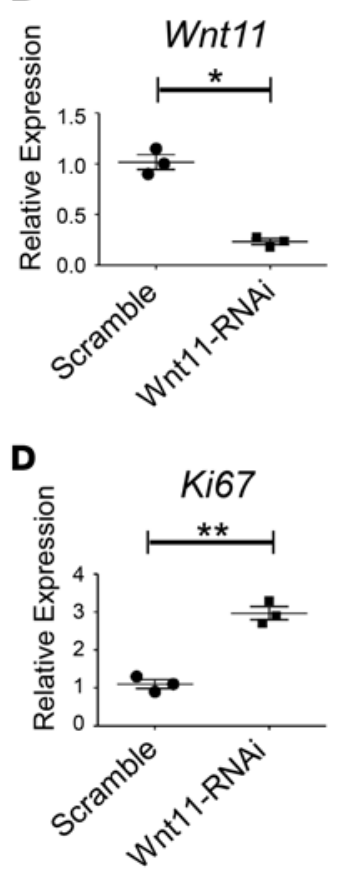

G

Scramble Wnt11-RNAi

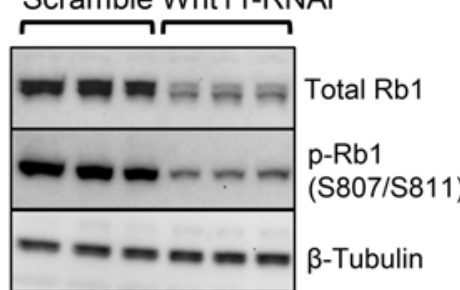

Neonatal Ventricular Myocytes (NRVMs)
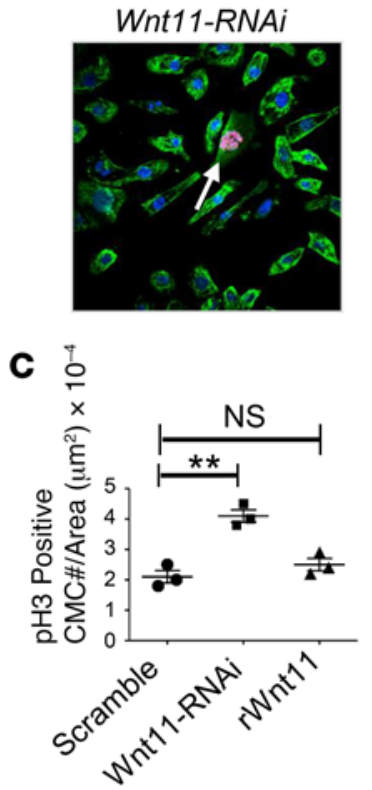

$\mathbf{E}$

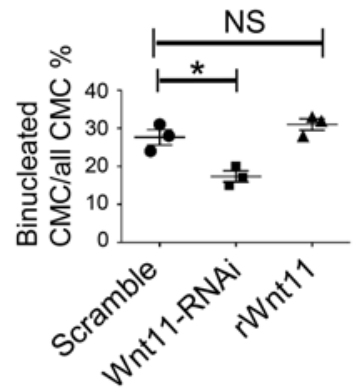

$\mathbf{F}$

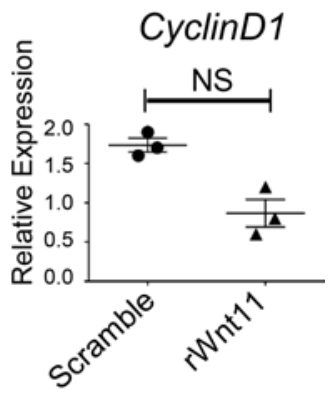

Figure 7. Wnt11 regulates neonatal ventricular myocyte proliferation. (A) Manipulation of Wnt11 in neonatal rat ventricular myocytes (NRVMs). Immunohistochemical (IHC) analysis of control (untreated), Wnt11-RNAi-treated, and exogenous recombinant Wnt11 (rWnt11)-treated NRVMs illustrates elongated mononucleated and phospho-histone H3-positive (p-H3-positive) cardiomyocytes (arrow) in Wnt11-suppressed NRVMs, and triangle-shaped, binucleated cardiomyocytes (arrow heads) in exogenous rWnt11-treated NRVMs. Original magnification, $\times 60$. (B) Wnt11 inhibition efficiency analysis (qRT-PCR) is shown (right). (C) p-H3-positive cell number/area $\left(\mu \mathrm{m}^{2}\right)$ in scramble-treated, Wnt11 siRNA-treated, or rWnt11-treated NRVMs.

(D) Expression analysis of proliferation marker (Ki67) using RNA from scramble-treated or Wnt11 siRNA-treated NRVMs (qRT-PCR). (E) Binucleation index (binucleated cardiomyocyte number $\times 100 /$ total cardiomyocytes/area $\left.\left[\mu \mathrm{m}^{2}\right]\right)$ analysis of scramble-treated, Wnt11 siRNA-treated, or rWnt11-treated NRVMs. $n=3$ replicates per condition. Data are representative of 3 independent experiments. (F) Expression of proliferation marker (cyclin D1) using RNA from scramble-treated or rWnt11-treated NRVMs (qRT-PCR). (C) Western analysis of Rb1 protein abundance and phosphorylation (S807/S811) in neonatal myocytes in response to Wnt11 inhibition suggests that Wnt11 loss is associated with Rb1 suppression and reduced p-Rb1. (H) Western analysis of JNK and PKC $\alpha$ protein expression and their phosphorylated forms $p$-JNK and $\mathrm{p}-\mathrm{PKC} \alpha$ in NRVMs in response to Wnt11 suppression. ${ }^{*} P$ $\leq 0.05$; ${ }^{*} P \leq 0.01$ by 1 -way ANOVA and posthoc Kruskal-Wallis test (C and $\mathbf{E}$ ) and 2-tailed Student's $t$ test (B, D, and $\mathbf{F}$ ) were used for intergroup analyses. NS, not significant.

role in survival and maintenance of cardiac stem/progenitor cells and regeneration of adult heart. Further, previous studies have shown that systemic hypoxia induces neonatal CMC proliferation and regeneration. Therefore, chamber-specific Wnt11 expression and its suppression under hypoxic conditions may implicate its role in chamber-specific regenerative capacity and pathological response to ischemic injury or hypoxic stress.

In this study, we focused on neonatal CMC proliferation. However, other potential Wnt11 functions in neonatal heart adaptation to hypoxic stress may involve fibroblasts, endothelium, or other non-myocytes in heart. Further, Wnt11 function may involve other biological processes such as apoptosis, fibrosis, angiogenesis, and extracellular matrix remodeling in heart. These are very intriguing and important determinants that need be investigated under normal and hypoxic conditions in combination with targeted genome editing in animal models, and in a larger patient cohort. These insights will be necessary for potential translational considerations in CHDs. 
A

Human

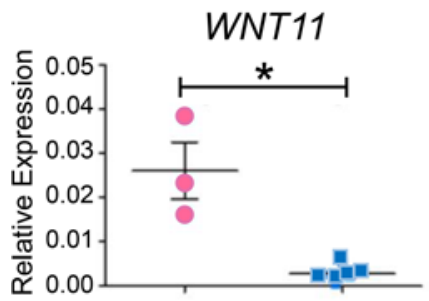

TOF O2 Saturation $>95 \%$

TOF O2 Saturation $<85 \%$
B

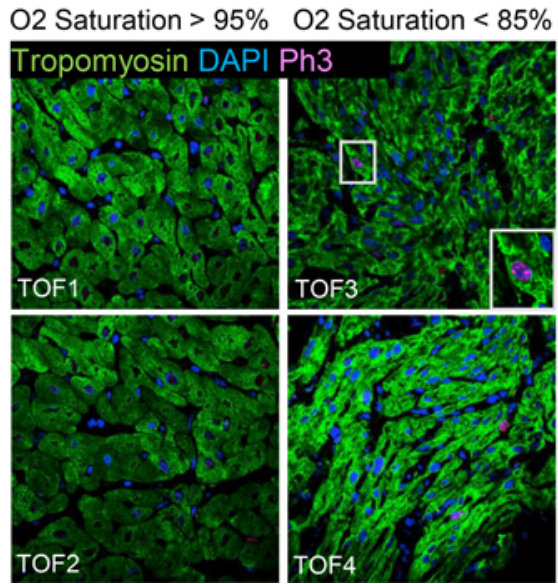

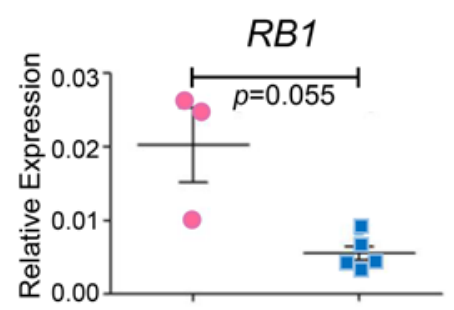

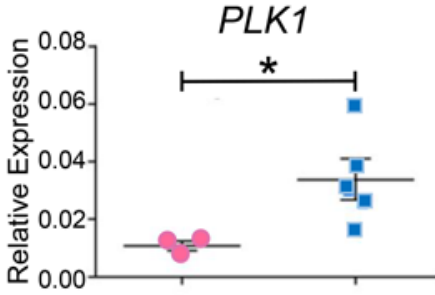

Figure 8. Wnt11 loss in hypoxemic TOF infants is associated with $R$ b1 suppression and induction of Plk1. (A) Expression of Wnt11, Rb1, and Plk1 in TOF patients (qRT-PCR), $n=5$ TOF cases were hypoxemic $\left(\mathrm{O}_{2}\right.$ saturation $\left.<85 \%\right)$, and $n=3$ cases with normal $\mathrm{O}_{2}$ saturation (>95\%). (B) Representative confocal images of anti-phospho-histone $\mathrm{H} 3$ (anti-p-H3) immunohistochemistry (IHC) in TOF infants. Arrows indicate representative $\mathrm{p}-\mathrm{H} 3-$ positive cardiomyocytes. Original magnification, $\times 60$. Note: The studies presented in this figure were performed in a new group of TOF patients that were not included in the studies presented in Figure 4 . Error bars represent SEM. ${ }^{*} P \leq 0.05$ by unpaired, 2-tailed Student's $t$ test.

\section{Methods}

Experimental animals. The experiments were conducted as part of an ongoing study under an active animal protocol approved by the Institutional Animal Care and Use Committee of UCLA. Animal handling and care followed the standards of the Guide for the Care and Use of Laboratory Animals. Pathogen-free male and female C57BL/6J mice from the inbred C57BL/6 strain were obtained from Charles River Laboratories. After establishment of breeding groups, successful mating was confirmed by plug detection. Timed pregnant dams were kept in cages under a 12-hour light/dark regime with food and water ad libitum. At E19.5, caesarian section delivery was performed and the hearts from male pups were excised to achieve P0 time point (as specified below). The remaining litters were allowed to deliver normally. Male pups and their mothers were kept in the same condition until the predecided specific end point.

Time points. Male pup hearts were excised at 3 time points of perinatal circulatory transition: P0 (birth before the ductal closure), P3 (establishing postnatal circulation), and P7 (terminal differentiation of the vast majority of CMCs based on current literature evidence) (on average $n=6$ male pups per biological replicate per time point were used). The RV and LV tissues were separated en bulk from each heart, leaving the septum out. The samples were snap frozen in liquid nitrogen and stored separately at $-80^{\circ} \mathrm{C}$.

Perinatal systemic hypoxia exposure. Neonatal pups were reared with their dams in either normoxia or hypoxia $\left(10 \% \mathrm{FIO}_{2}\right)$ using a BioSpherix OxyCycler normobaric hypoxia chamber and maintained until the predecided end points. The dams carrying the experimental group (hypoxia) were acclimatized by decreasing $\mathrm{FIO}_{2}$ by $2 \%$ daily for at least 5 days preceding the experiment starting at E14.5 to reach $10 \%$ on E19.5, as previously described with minor modification (20), and maintained with their offspring until the predefined time point (P0, $\mathrm{P} 3$, or P7), whereas the dams carrying the control neonates (normoxia) were maintained in a normal environment.

Perinatal Wnt11 knockdown in perinatal mouse using Vivo-Morpholino. Wnt11-specific antisense oligonucleotide (0.5 mM Wnt11 Vivo-Morpholino stock) was administered by 2 days of i.v. injections at $12.5 \mathrm{mg} /$ $\mathrm{kg}$. For a typical 20-g mouse the dose was approximately 25 nmoles $(50 \mu \mathrm{l})$ per injection. For a 2-g pup the dose was approximately 2.5 nmoles $(5 \mu 1)$ per injection. The sequences of negative control or Wnt11 Vivo-Morpholino are as follows: negative control, 5'-cctcttacctcagttacaatttata-3'; Wnt11, 5'-tgcaggagccgcaccgaactccgcc-3' (Gene Tools). 
RNA-Seq and bioinformatics analysis. In this study we used the mRNA expression data derived from deep RNA-Seq data sets of male C57B/6 mouse LVs and RVs at P0 (before the ductal closure), P3 (transition), and P7 (terminal differentiation of the vast majority of CMCs). Data preprocessing, RNA-Seq, and bioinformatics analysis steps follow exactly the procedure we previously described (1). The RNA-Seq data were previously deposited in the NCBI's Gene Expression Omnibus repository under Neonatal Heart Maturation SuperSeries GSE85728 (http://www.ncbi.nlm.nih.gov/geo/query/acc.cgi?acc=GSE85728) PMID: 27591185 (1).

WGCNA. Genes with mean reads per kilobase of transcript per million mapped reads (RPKM) greater than or equal to 3 in at least one sample ( 3 replicates) of each category and variation greater than or equal to 0.2 across samples were included to construct the signed network from LV and RV data sets separately. Soft power parameter was estimated and used to derive a pairwise distance matrix for selected genes. Based on the resulting adjacency matrix, we calculated the topological overlap, a concept defined for weighted networks by Zhang and Langfelder (47), which is a robust and biologically meaningful measure of network interconnectedness (that is the strength of 2 genes' coexpression relationship with respect to all other genes in the network). Using the R package, gene modules were constructed as groups of genes with highly similar coexpression relationships by performing average linkage hierarchical clustering on the topological overlap. The dynamic hybrid tree cut algorithm was used to cut the hierarchical clustering tree and define the modules as branches (clusters) from the tree cutting as previously described (48). The average expression profile of each module was summarized and presented as the first principal component (referred to as module eigengene). Modules whose eigengenes were highly correlated were merged. Modules were detected independently in LVs and RVs and the correlation between a module and a trait (P0, P3, or P7) was calculated. Unique stage-specific modules in LVs and RVs were defined as those with correlation coefficient $r$ greater than or equal to 0.7 and $P$ value less than or equal to 0.05 between the module eigengene and the maturation stage (P0, P3, or P7). Functional enrichment was determined by gene ontology analysis using database for annotation, visualization, and integrated discovery (DAVID). Pathway analysis of the expression data was done with QIAGEN's Ingenuity Pathway Analysis (IPA), www.qiagen.com/ingenuity. Pathways with FDR $P$ less than or equal to 0.05 (Benjamini-Hochberg method) were found to be significantly differentially expressed.

Module preservation analysis. Preservation between LV- and RV-WGCNA-derived modules was examined. The module overlap was evaluated using Fisher's exact test for each pair of LV/RV modules and $Z$-score summary plots were generated. $Z$ summary less than 2 indicates that modules were weakly or not preserved, $Z$ summary between 2 and 10 indicates that modules were moderately preserved, and $Z$ summary greater than 10 indicates that modules were well or strongly preserved.

Other bioinformatic and computational methods. The statistical significance for differential gene expression was assessed by Fisher's exact test. Pearson's correlation coefficients for gene expression were calculated in R. Principal component analysis (PCA) was conducted using R function prcomp. The top 1,000 varied mRNAs based on Tophat alignment results were used to generate PCAs. The heatmap function of R, which employs a hierarchical cluster algorithm, was used to draw heatmap figures. The log2-transformed data were preprocessed by median centering of the data for each set and then hierarchically clustered using centered correlation as the similarity metric and average linkage as the clustering method.

Validation of gene expression by $q R T-P C R$. Total RNA was isolated from pooled male pups' LV free walls and RV free walls separately, using an RNeasy Mini Kit (QIAGEN), according to the manufacturer's protocol. DNA contamination was eliminated using an RNase-Free DNase Set (QIAGEN), according to the manufacturer's instructions. For reverse transcription, $1 \mu \mathrm{g}$ of total RNA was used to generate first-strand cDNA with oligo-dT primers. Real-time PCR was performed using the SYBR Green Mix (Bio-Rad) on the CFX96 Real-time System (Bio-Rad). qRT-PCR was performed using housekeeping genes GAPDH, S26, or $\mathrm{B} 2 \mathrm{M}$ for normalization and significant differences in gene expression were reported based on fold change. Primer sequences of validated mRNAs are listed in Table 1.

Immunohistochemistry. Cells were fixed in $4 \%(\mathrm{v} / \mathrm{v})$ formaldehyde in PBS for 20 minutes. After rinsing with PBS, the cells were incubated with $0.1 \%$ Triton X-100 in PBS for 15 minutes at room temperature and rinsed. Heart tissue was fixed in $4 \%(\mathrm{v} / \mathrm{v})$ formaldehyde, embedded into paraffin, and cut into 5- $\mu \mathrm{m}$-thick tissue sections. After deparaffinization, slides were subjected to antigen retrieval. After blocking in PBS containing 10\% bovine serum albumin for 1 hour, the cells and tissue sections were incubated with primary antibodies overnight and then appropriate AlexaFluor-conjugated secondary antibodies (Life Technologies) for 1 hour. Cell nuclei were eventually counterstained by DAPI in the mounting medium (Life Technologies). Images were recorded on a Nikon confocal microscope. To 
Table 1. List of primers used for quantitative RT-PCR

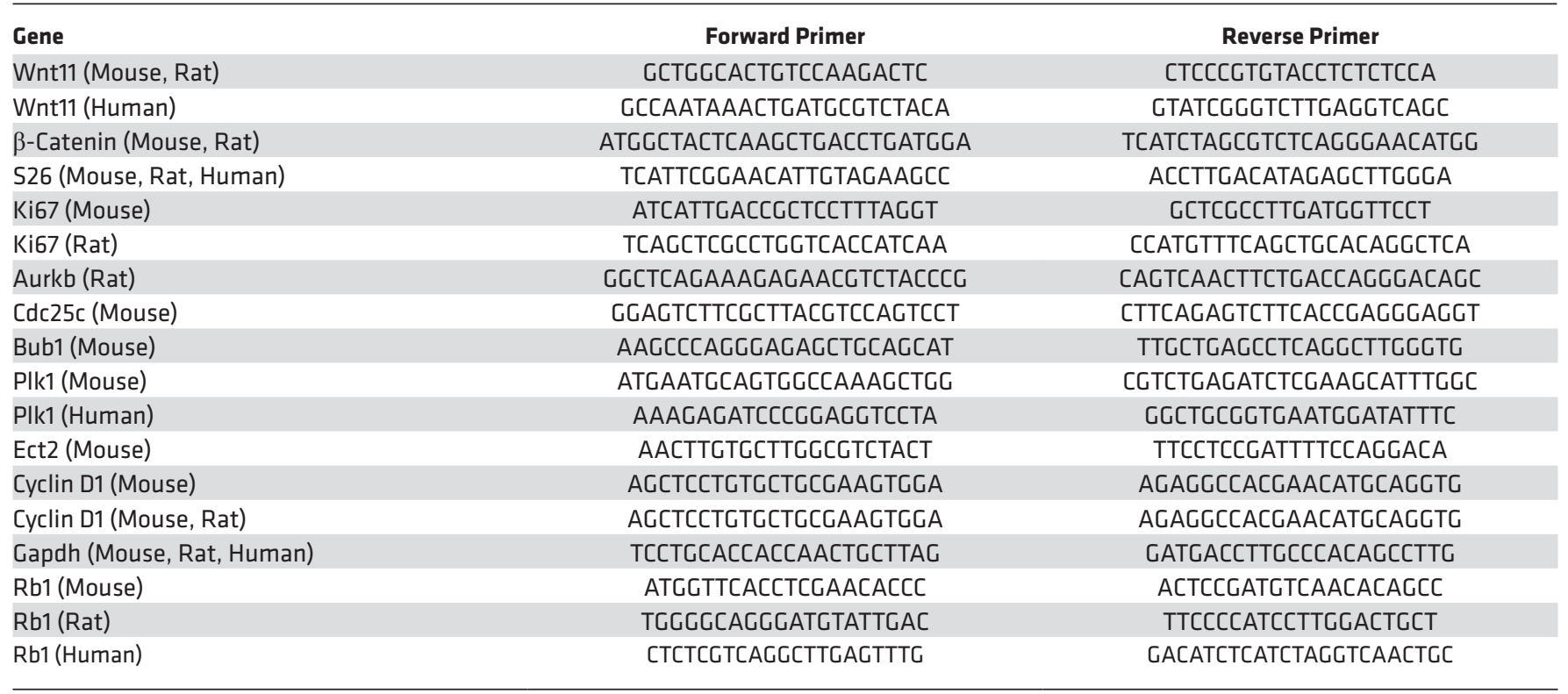

count p-H3-positive CMCs, we carefully examined the sections under confocal microscopy to ensure the counted p-H3-positive nuclei were fully imbedded in sarcomeres as expected for CMCs. The binucleation index was increased in rWnt11-treated cells (Figure 6C), measured after excluding all p-H3positive CMCs in this analysis in order to determine the accumulated effect of karyokinesis without following cytokinesis as a hallmark of neonatal CMC maturation. Primary antibodies used to detect the expression of the proteins of interest are listed in Table 2.

Western blot analysis. After protein quantification, protein samples were electrophoresed in $4 \%-12 \%$ SDS polyacrylamide gels before transfer to PVDF membranes (Millipore). HRP-conjugated secondary antibodies (Bio-Rad) were used followed by ECL reaction to develop the blots according to the manufacturer's instructions. Band intensities from film were analyzed by IMAGEQUANT 5.2 software (Molecular Dynamics) if needed. Primary antibodies used to detect the expression of the proteins of interest are listed in Table 2.

NRVM culture, $r W n t 11$, and Wnt11 siRNA treatment. NRVMs were isolated from 2-day-old rat pups and cultured in DMEM/5\% (vol/vol) FBS with antibiotics at $37^{\circ} \mathrm{C}$ and $5 \% \mathrm{CO}_{2}$. On the next day, cells were incubated with $200 \mathrm{ng} / \mathrm{ml} \mathrm{rWnt} 11$ (R\&D Systems) for 48 hours. For Wnt11 siRNA treatment, NRVMs were transfected in 6-well plates with $30 \mathrm{nM}$ siRNA and $6 \mu$ Lipofectamine RNAiMAX Transfection Reagent (Life Technologies) in Opti-MEM (Life Technologies). Culture medium was changed to DMEM/5\% FBS after 4 hours. After 48 hours, total RNA was obtained using TRIzol reagent (Life Technologies) and total protein was isolated using cell lysis buffer (Life Technologies). For identification of phosphorylated proteins, Phosphatase Inhibitor Cocktail (Sigma-Aldrich) was added to the lysis buffer (Table 3).

Human studies. Pediatric patients with clinical diagnosis of a CHD were enrolled in the Congenital Heart Defect Bio-banking core (the CHD-BioCore) using a protocol approved by the UCLA Institutional Review Board. Written informed consents were obtained from all participants. Infants with isolated (nonsyndromic) TOF, VSD, or DORV and normal primary genetic screening results, including karyotype to rule out chromosomal abnormalities, florescence in situ hybridization (FISH) to rule out Di George Syndrome, and SNP microarray to rule out copy number variations were selectively included in this study. Human heart specimens from the RVOT were collected during clinically indicated cardiac operations. The specimens were immediately snap frozen using liquid nitrogen. Total RNAs were isolated using standard methods. Quantitative expression array (RT-PCR array) was performed in the UCLA GenoSeq Core. The housekeeping gene GAPDH was used for normalization. The expression data are presented as a heatmap to visualize the pattern of expression. Wnt gene expression (RNA-Seq data) were obtained from $\mathrm{M}$. Touma's unpublished observations. Pearson's correlation, linear regression module, was implemented for correlation studies and a $P$ value less than or equal to 0.05 was considered significant. 
Table 2. List of antibodies and their sources

\begin{tabular}{cc}
\hline Gene & Antibodies (Source) \\
\hline Wnt11 & OriGene, TA308507 \\
$\beta$-Catenin & Zymed, 71-2700 \\
\hline p- $\beta$-Catenin S675, S552 & Cell Signaling, 4176, 5651 \\
Rb1 & Abcam, ab181616 \\
\hline p-Rb1 (S807/811) & Cell Signaling, 8516 \\
\hline p-Rb1 (S780) & Cell Signaling, 8180 \\
Cyclin D1 & Invitrogen, MA5-14512 \\
P21 & Santa Cruz Biotechnology, sc-397 \\
\hline p-P21 & Santa Cruz Biotechnology, sc-20220-r \\
\hline JNK & Santa Cruz Biotechnology, sc-571 \\
\hline p-JNK & Santa Cruz Biotechnology, sc-12882 \\
PKC $\alpha$ & Cell Signaling, 2056 \\
p-PKC $\alpha$ & Cell Signaling, 9375 \\
\hline p-Histone H3 (Ser10) & Millipore, 06-570 \\
\hline$\beta-$ Actin & Thermo Fisher Scientific, MA5-15739 \\
\hline
\end{tabular}

Echocardiography. To evaluate cardiac function of neonatal pups, a Vevo2100 high-frequency probe $(30 / 45 \mathrm{MHz}$, Visual Sonics) was used as previously described $(49,50)$. Briefly, ultrasound images were obtained in the transverse and sagittal planes for each pup. Scanning B-mode images were used to identify basic cardiac structures. LV and $\mathrm{RV}$ chambers were annotated using the longitudinal 4-chamber view. M-mode cardiac scanning was performed using the $30-\mathrm{MHz}$ transducer. M-mode images were obtained from the short-axis view and were further analyzed to measure ventricular wall thickness and chamber dimensions and to calculate percentage ejection fraction. Temporal changes between LV end-systolic dimension (LVESD) and LV end-diastolic dimension (LVEDD) throughout the cardiac cycle were used for the calculation of percentage fractional shortening as follows: percentage fractional shortening $=($ LVESD $/$ LVEDD $) \times 100$.

Primer design. Primer sequences of validated mRNAs are listed in Table 1.

Antibodies and other reagents. Primary antibodies used to detect the expression of the proteins of interest are listed in Table 2. Other resources are listed in Table 3.

Statistics. Quantified results are presented as mean \pm SEM. Student's $t$ test (unpaired, 2-tailed) and ANOVA with post-hoc Kruskal-Wallis were used for comparing 2 groups and more than 2 groups, respectively; $P$ less than or equal to 0.05 was considered significant, unless specified otherwise. The correlation of gene expression for each $\mathrm{mRNA} /$ trait pair was calculated using Pearson's correlation and Benjamini-Hochberg correction methods. A Benjamini-Hochberg-adjusted correlation $P$ value less than or equal to 0.05 was considered significant.

Study approval. All animal-related experimental protocols were approved by the UCLA Animal Care and Use Committee.

All human studies were conducted in accordance with regulation of the UCLA Institutional Review Board. Patients' parents/legal guardians provided written informed consent prior to their participation in the study.

\section{Author contributions}

MT conceived the project, designed and performed the research, analyzed most of the data, managed funding, and wrote the manuscript. YW managed funding and participated in experimental design and manuscript writing. XK and YZ performed experiments and participated in data analysis and manuscript writing. AAC, FG, XX, and GC supported bioinformatics analysis. RB and BR contributed human samples and participated in data analysis. ME supported in vivo hypoxia exposure experiments.

\section{Acknowledgments}

This work was supported by grants from the NIH/Child Health Research Center (5K12HD034610/K12) and the UCLA-Children's Discovery Institute and Today and Tomorrow Children's Fund to M. Touma; NIH/ Predoctoral training grant (T90DE022734) to A.A. Cass; R01HG006264 to X. Xiao; HL070079, HL103205, HL108186 and HL110667, and UCLA-CT-

SI-Cardiovascular Pilot Team Research Grant UL1TR000124 to Y. Wang. We acknowledge the support of the NINDS Informatics Center for Neurogenetics and Neurogenomics grant (P30 NS062691) to G. Coppola and F. Gao.

Address correspondence to: Marlin Touma, David Geffen School of Medicine, University of California, Los Angeles, 10833 Le Conte Avenue, B2-375, MDCC, Los Angeles, California 90095, USA. Phone: 310.206.6197; Email: mtouma@mednet.ucla.edu.
Table 3. List of other reagents and their sources

\begin{tabular}{|c|c|}
\hline Reagent & Source \\
\hline Rat Wnt11 siRNA & QIAGEN, SI01604428 \\
\hline Scramble siRNA & Bioland Scientific \\
\hline $\begin{array}{l}\text { Antisense Oligo Nucleotide (Vivo- } \\
\text { Morpholino-Wnt11) }\end{array}$ & Gene Tools \\
\hline Scramble Morpholino & Gene Tools \\
\hline Recombinant Human Wnt11 & R\&D Systems, 6179-WN \\
\hline Phosphatase Inhibitors Cocktail & Cocktail, Sigma-Aldrich \\
\hline
\end{tabular}


1. Touma M, et al. Decoding the long noncoding RNA during cardiac maturation: a roadmap for functional discovery. Circ Cardiovasc Genet. 2016;9(5):395-407.

2. Finnemore A, Groves A. Physiology of the fetal and transitional circulation. Semin Fetal Neonatal Med. 2015;20(4):210-216.

3. Sinha SK, Donn SM. Fetal-to-neonatal maladaptation. Semin Fetal Neonatal Med. 2006;11(3):166-173.

4. Rudolph AM. Myocardial growth before and after birth: clinical implications. Acta Paediatr. 2000;89(2):129-133.

5. Smolich JJ, Walker AM, Campbell GR, Adamson TM. Left and right ventricular myocardial morphometry in fetal, neonatal, and adult sheep. Am J Physiol. 1989;257(1 Pt 2):H1-H9.

6. Porrello ER, et al. Transient regenerative potential of the neonatal mouse heart. Science. 2011;331(6020):1078-1080.

7. Nakada Y, Kimura W, Sadek HA. Defining the limit of embryonic heart regeneration. Circulation. 2015;132(2):77-78.

8. Muralidhar SA, Mahmoud AI, Canseco D, Xiao F, Sadek HA. Harnessing the power of dividing cardiomyocytes. Glob Cardiol Sci Pract. 2013;2013(3):212-221.

9. Mahmoud AI, Porrello ER, Kimura W, Olson EN, Sadek HA. Surgical models for cardiac regeneration in neonatal mice. Nat Protoc. 2014;9(2):305-311.

10. Sen S, Sadek HA. Neonatal heart regeneration: mounting support and need for technical standards. J Am Heart Assoc. 2015;4(1):e001727.

11. Mahmoud AI, et al. Meis1 regulates postnatal cardiomyocyte cell cycle arrest. Nature. 2013;497(7448):249-253.

12. Tane S, Ikenishi A, Okayama H, Iwamoto N, Nakayama KI, Takeuchi T. CDK inhibitors, p21(Cip1) and p27(Kip1), participate in cell cycle exit of mammalian cardiomyocytes. Biochem Biophys Res Commun. 2014;443(3):1105-1109.

13. Hirai M, Chen J, Evans SM. Tissue-specific cell cycle indicator reveals unexpected findings for cardiac myocyte proliferation. Circ Res. 2016;118(1):20-28.

14. McFadden DG, Barbosa AC, Richardson JA, Schneider MD, Srivastava D, Olson EN. The Hand1 and Hand2 transcription factors regulate expansion of the embryonic cardiac ventricles in a gene dosage-dependent manner. Development. 2005;132(1):189-201.

15. Lien CL, Wu C, Mercer B, Webb R, Richardson JA, Olson EN. Control of early cardiac-specific transcription of Nkx2-5 by a GATA-dependent enhancer. Development. 1999;126(1):75-84.

16. Fishman NH, Hof RB, Rudolph AM, Heymann MA. Models of congenital heart disease in fetal lambs. Circulation. 1978;58(2):354-364.

17. Bhat V, Belaval V, Gadabanahalli K, Raj V, Shah S. Illustrated imaging essay on congenital heart diseases: multimodality approach part III: cyanotic heart diseases and complex congenital anomalies. J Clin Diagn Res. 2016;10(7):TE01-TE10.

18. Zabala LM, Guzzetta NA. Cyanotic congenital heart disease (CCHD): focus on hypoxemia, secondary erythrocytosis, and coagulation alterations. Paediatr Anaesth. 2015;25(10):981-989.

19. Kimura W, Sadek HA. The cardiac hypoxic niche: emerging role of hypoxic microenvironment in cardiac progenitors. Cardiovasc Diagn Ther. 2012;2(4):278-289.

20. Nakada Y, et al. Hypoxia induces heart regeneration in adult mice. Nature. 2017;541(7636):222-227.

21. Olson EN, Srivastava D. Molecular pathways controlling heart development. Science. 1996;272(5262):671-676.

22. Theis JL, et al. Compound heterozygous NOTCH1 mutations underlie impaired cardiogenesis in a patient with hypoplastic left heart syndrome. Hum Genet. 2015;134(9):1003-1011.

23. Dietrich AC, Lombardo VA, Veerkamp J, Priller F, Abdelilah-Seyfried S. Blood flow and Bmp signaling control endocardial chamber morphogenesis. Dev Cell. 2014;30(4):367-377.

24. Pandur P, Maurus D, Kühl M. Increasingly complex: new players enter the Wnt signaling network. Bioessays. 2002;24(10):881-884.

25. Bisson JA, Mills B, Paul Helt JC, Zwaka TP, Cohen ED. Wnt5a and Wnt11 inhibit the canonical Wnt pathway and promote cardiac progenitor development via the Caspase-dependent degradation of AKT. Dev Biol. 2015;398(1):80-96

26. He Z, et al. Transduction of Wnt11 promotes mesenchymal stem cell transdifferentiation into cardiac phenotypes. Stem Cells Dev. 2011;20(10):1771-1778.

27. Cohen ED, Miller MF, Wang Z, Moon RT, Morrisey EE. Wnt5a and Wnt11 are essential for second heart field progenitor development. Development. 2012;139(11):1931-1940.

28. Sinha T, et al. Mapping the dynamic expression of Wnt11 and the lineage contribution of Wnt11-expressing cells during early mouse development. Dev Biol. 2015;398(2):177-192.

29. Zhou W, et al. Modulation of morphogenesis by noncanonical Wnt signaling requires ATF/CREB family-mediated transcriptional activation of TGFbeta2. Nat Genet. 2007;39(10):1225-1234.

30. Mazzotta S, Neves C, Bonner RJ, Bernardo AS, Docherty K, Hoppler S. Distinctive roles of canonical and noncanonical Wnt signaling in human embryonic cardiomyocyte development. Stem Cell Reports. 2016;7(4):764-776.

31. Nagy II, et al. Wnt-11 signalling controls ventricular myocardium development by patterning N-cadherin and beta-catenin expression. Cardiovasc Res. 2010;85(1):100-109.

32. Abdul-Ghani M, Dufort D, Stiles R, De Repentigny Y, Kothary R, Megeney LA. Wnt11 promotes cardiomyocyte development by caspase-mediated suppression of canonical Wnt signals. Mol Cell Biol. 2011;31(1):163-178.

33. Flaherty MP, Dawn B. Noncanonical Wnt11 signaling and cardiomyogenic differentiation. Trends Cardiovasc Med. 2008;18(7):260-268.

34. Roderick HL, Cook SJ. Ca ${ }^{2+}$ signalling checkpoints in cancer: remodelling $\mathrm{Ca}^{2+}$ for cancer cell proliferation and survival. Nat Rev Cancer. 2008;8(5):361-375.

35. Panáková D, Werdich AA, Macrae CA. Wnt11 patterns a myocardial electrical gradient through regulation of the L-type $\mathrm{Ca}(2+)$ channel. Nature. 2010;466(7308):874-878.

36. Sim CB, et al. Dynamic changes in the cardiac methylome during postnatal development.FASEB J. 2015;29(4):1329-1343

37. An CI,Ichihashi Y,Peng J, Sinha NR, Hagiwara N. Transcriptome dynamics and potential roles of Sox6 in the postnatal heart.PLoS One. 2016;11(11):e0166574.

38. Sdek $\mathrm{P}$, et al. $\mathrm{Rb}$ and $\mathrm{p} 130$ control cell cycle gene silencing to maintain the postmitotic phenotype in cardiac myocytes. $J$ Cell 
Biol. 2011;194(3):407-423.

39. Angelis E, Zhao P, Zhang R, Goldhaber JI, Maclellan WR. The role of E2F-1 and downstream target genes in mediating ischemia/reperfusion injury in vivo. J Mol Cell Cardiol. 2011;51(6):919-926.

40. MacLellan WR, et al. Overlapping roles of pocket proteins in the myocardium are unmasked by germ line deletion of p130 plus heart-specific deletion of Rb. Mol Cell Biol. 2005;25(6):2486-2497.

41. Morishita Y, et al. Wnt11 Gene Therapy with adeno-associated virus 9 improves recovery from myocardial infarction by modulating the inflammatory response. Sci Rep. 2016;6:21705.

42. Qiu Z, Ghosh A. A calcium-dependent switch in a CREST-BRG1 complex regulates activity-dependent gene expression. Neuron. 2008;60(5):775-787.

43. Lange C, Mix E, Rateitschak K, Rolfs A. Wnt signal pathways and neural stem cell differentiation. Neurodegener Dis. 2006;3(1-2):76-86.

44. Lima L, et al. Reference genes for addressing gene expression of bladder cancer cell models under hypoxia: a step towards transcriptomic studies. PLoS One. 2016;11(11):e0166120.

45. Katoh M. WNT/PCP signaling pathway and human cancer (review). Oncol Rep. 2005;14(6):1583-1588.

46. Gessert S, Kühl M. The multiple phases and faces of wnt signaling during cardiac differentiation and development. Circ Res. 2010;107(2):186-199.

47. Langfelder P, Horvath S. WGCNA: an R package for weighted correlation network analysis. BMC Bioinformatics. 2008;9:559

48. Langfelder P, Zhang B, Horvath S. Defining clusters from a hierarchical cluster tree: the Dynamic Tree Cut package for R. Bioinformatics. 2008;24(5):719-720.

49. Nakashima Y, et al. Nkx2-5 suppresses the proliferation of atrial myocytes and conduction system. Circ Res. 2014;114(7):1103-1113.

50. Ganguly A, Touma M, Thamotharan S, De Vivo DC, Devaskar SU. Maternal calorie restriction causing uteroplacental insufficiency differentially affects mammalian placental glucose and leucine transport molecular mechanisms. Endocrinology. 2016;157(10):4041-4054. 